\title{
Multigrid Convergent Principal Curvature Estimators in Digital Geometry ${ }^{1}$
}

\author{
David Coeurjolly ${ }^{\mathrm{a}}$, Jacques-Olivier Lachaud ${ }^{\mathrm{b}, \mathrm{c}}$, Jérémy Levallois ${ }^{\mathrm{a}, \mathrm{b}, *}$ \\ ${ }^{a}$ Université de Lyon, CNRS, INSA-Lyon, LIRIS, UMR5205, F-69621, France \\ ${ }^{b}$ Université de Savoie, CNRS, LAMA, UMR 5127, F-73776, France \\ ${ }^{c}$ Université Grenoble-Alpes, CNRS, LJK, UMR 5224, F-38041, France
}

\begin{abstract}
In many geometry processing applications, the estimation of differential geometric quantities such as curvature or normal vector field is an essential step. In this paper, we investigate a new class of estimators on digital shape boundaries based on Integral Invariants. More precisely, we provide both proofs of multigrid convergence of principal curvature estimators and a complete experimental evaluation of their performances.
\end{abstract}

Keywords: Digital geometry, curvature estimation, multigrid convergence, integral invariants.

\section{Introduction}

Context and objectives. In many shape processing applications, the estimation of differential quantities on the shape boundary is usually an important step. Their correct estimation makes easier further processing, like quantitative evaluation, feature detection, shape matching or visualization. This paper focuses on estimating the curvature tensor on the boundary of digital shapes. Such digital structures are subsets of the 3-dimensional digital space $\mathbb{Z}^{3}$ and come generally from the digitization of some Euclidean shape. Of course, the curvature tensor estimation should be as close as possible to the curvature tensor of the underlying Euclidean shape before digitization. Digital data form a special case of discrete data with specific properties: (1) digital data cannot sample the boundary of the Euclidean shape (i.e. they do not lie on the shape boundary), (2) digital data are distributed around the true sample according to arithmetic noise, which looks rather uniform over a range $[-h, h]$ from a statistical point of view, where $h$ is the digitization grid step. Another way of stating these characteristics is to say that the Hausdorff distance between the Euclidean shape and its digitization is some $O(h)$. Of course, the quality of the estimation should be improved as the digitization step gets finer and finer. This property is called the multigrid convergence $[1,2]$. It is similar in spirit with the stability property in Geometry processing: given a continuous shape and a specific sampling of if boundary, the estimated measure should converge to the Euclidean one when the sampling become denser (e.g. [3, 4]).

\footnotetext{
${ }^{*}$ Corresponding author.
} 
Our objective is to design a curvature tensor estimator for digital data such that: (1) it is provably multigrid convergent, (2) it is accurate in practice, (3) it is computable in an exact manner, (4) it can be efficiently computed either locally or globally (evaluation at a single surface point or extraction of the curvature tensor field), (5) it is robust to further perturbations (like bad digitization around the boundary, outliers).

Related works for meshes. Digital data being discrete in nature, it is interesting to look at the curvature estimation techniques on triangulated meshes. In computer graphics and geometry processing, there exists a vast family of techniques to estimate either the mean or Gaussian curvatures, or sometimes the full curvature tensor. Most of them are local (i.e. limited to a 1-ring or 2-ring of neighbors) but exhibit correct results for nice meshes. They generally fall into three categories: fitting, discrete methods, curvature tensor estimation. We may refer to [5] and [6] for comprehensive evaluations, and Desbrun et al. [7] or Bobenko and Suris [8] for a more general theory. Most of them have not theoretical convergence guarantees even without noise on the mesh. We may quote [9] and [10] as approaches trying to tackle perturbation through averaging.

For Gaussian curvature estimated with Gauss-Bonnet approach (angle defect), Xu [11] provides a stability theorem for triangulated mesh whose vertices lie on the underlying smooth manifold, with valence 6 and parallelogram condition (each 1-ring of neighbors is projected as a parallelogram onto a plane). Assuming a sampling with density $\delta$, he provides an additional convergence property whenever the sampling is perturbated by some $O\left(\delta^{\alpha}\right)$, but $\alpha>2$ (inadequate for discrete data). Note that should the triangulated mesh not satisfy these requirements, then such estimation does not converge.

The integral measures of curvatures, based on normal cycle theory $[12,13]$ is another notable approach for estimating curvature information on a triangulated mesh. Authors exhibit some convergence results for triangulated meshes with vertices lying on the underlying smooth Euclidean shape boundary. In this case, if the mesh has Hausdorff distance to shape boundary below $\epsilon$, convergence is obtained with speed/error $O(\epsilon)$ under some hypotheses.

Finally, in geometry processing, interesting mathematical tools have been developed to design differential estimators on smooth surfaces based on integral invariants [14, 15]. They consist in moving a kernel along the shape surface and in computing integrals on the intersection between the shape and the kernel. Authors have demonstrated that some integral quantities provide interesting curvature information when the kernel size tends to zero. They also achieve stability depending on the kernel radius and on $\epsilon$, for instance in the case of a mesh sampling.

Related works for point clouds. When having only discrete data (i.e. a cloud of points), the most natural way to approach curvature(s) is to fit a polynomial surface of degree two at least. Perhaps the best representative of these techniques is the osculating jets of Cazals and Pouget [16]. The authors provide $O\left(\delta^{2}\right)$ convergence results when data is a surface sampling, assuming $\delta$ is the density of points. There is no 
theoretical result in presence of noise, although the least-square fitting of osculating jets is very robust to noise in practice.

Another family of techniques exploit the Voronoi diagram [17, 18, 4]. The idea behind these approaches is, instead of fitting the tangent space, to estimate at best the orthogonal space. The convolved covariance measure introduced by Mérigot et al. [4] is particularly appealing since this measure achieves robustness even for arbitrary compact sets, essentially in $O(\sqrt{\epsilon})$. It is in some sense an integral measure of the covariance matrix of the normal cone around the point of interest. However, convergence of curvature(s) is subject to several parameters $r$ and $R$ which contribute contradictorily to the Hausdorff error. In practice, this approach gives results comparable to osculating jets for curvatures.

Recently, several authors have developed new interesting approaches for estimating the normal vector field on noisy point of clouds, even in the presence of sharp features [19], [20], [21]. Furthermore, Boulch and Marlet [20] gives probabilistic convergence results. Although they cannot be used as is for curvature computation, they could be used in parallel with curvature estimation techniques to locate sharp features in a first pass, and to limit curvature estimations to smooth zones.

Related works for digital data. In Digital Geometry, we usually consider multigrid convergence as an essential criterion [2]. Hence, in dimension 2, parameter free convergence results have been obtained for length [22] and normal vector estimation [23]. Based either on binomial convolution principles [24, 25], or polynomial fitting [26], convergence results can also be obtained for higher order derivatives of digital curves. Algorithms are parametrized by the size of the convolution or fitting kernel support and convergence theorem holds when such support size is an increasing function of the grid resolution and some shape characteristics.

For curvature estimation along 2D curves, multigrid convergence of parameter free estimator is still challenging, although accurate experimental results have been obtained with maximal digital circular arcs [27] and with global optimization [28]. In 3D digital space, several empirical methods exist for estimating curvatures, but none achieves multigrid convergence (e.g. see [29, 30]). In [31], we recently presented a digital estimator for mean curvature for $2 \mathrm{D}$ and $3 \mathrm{D}$ digital objects, which achieve multigrid convergence in $O\left(h^{\frac{1}{3}}\right)$.

Contributions. This paper completes [31] to propose a new curvature tensor estimator for digital data, which casts carefully the Integral Invariant (II) method of $[14,15]$ into the digital world. This estimator is a non-trivial extension of our mean digital curvature estimator [31], since it involves the compution of digital moments and covariance matrices, and requires results from matrix perturbation theory.

The contributions of the paper can be sketched as follows. First, we define digital versions of integral invariant estimators with multigrid convergence results (Theorems 3 and 4). We provide an explicit formula for the kernel size, which guarantees uniform convergence in $O\left(h^{\frac{1}{3}}\right)$ for smooth enough curves and surfaces (Theorem 6). Furthermore, we demonstrate that these estimators have simple, exact and efficient implementations (available in DGTAL library [32]). We provide an extensive comparative evaluation of these 
estimators (mean curvature, principal curvatures), which shows that they compete with classical ones in terms of accuracy (Section 4). Computation speed is also considered, and our method is for instance ten times faster than the osculating jets. Finally, we show empirical results illustrating the robustness to noise and outliers of our estimators.

\section{Preliminaries}

\subsection{Shapes, digital shapes and multigrid convergence}

Since we are interested in evaluating both theoretically and experimentally the behavior of a given differential estimator on digital object boundaries, we first have to formalize links between Euclidean objects and digital ones with the help of a digitization process. Let us consider a family $\mathbb{X}$ of smooth and compact subsets of $\mathbb{R}^{d}$. In Section 2.3 we will be more precise on the notion of smoothness for shapes $X \in \mathbb{X}$. We denote $\mathrm{D}_{h}(X)$ the digitization of $X$ in a $d$-dimensional grid of grid step $h$. More precisely, we consider classical Gauss digitization defined as

$$
\mathrm{D}_{h}(X) \stackrel{\text { def }}{=}\left(\frac{1}{h} \cdot X\right) \cap \mathbb{Z}^{d}
$$

where $\frac{1}{h} \cdot X$ is the uniform scaling of $X$ by factor $\frac{1}{h}$. Furthermore, the set $\partial X$ denotes the frontier of $X$ (i.e. its topological boundary). If $z \in \mathbb{Z}^{d}$, then $Q_{z}$ denotes the unit $d$-dimensional cube of $\mathbb{R}^{d}$ centered on $z$. The $h$-frontier $\Delta_{h} Z$ of a digital set $Z \subset \mathbb{Z}^{d}$ is defined as $\Delta_{h} Z \stackrel{\text { def }}{=} \partial\left(h \cdot \cup_{z \in Z} Q_{z}\right)$. Therefore, the $h$-frontier of $\mathrm{D}_{h}(X)$ is a $d$-1-dimensional subset of $\mathbb{R}^{d}$, which is close to $\partial X$. We will precise the term "close" later in this section. Since this paper deals with multigrid convergence, digital shapes will always come from the digitization of continuous ones. To simplify notations, the $h$-frontier of the Gauss digitization at step $h$ of a shape $X$ will simply be denoted by $\partial_{h} X \stackrel{\text { def }}{=} \Delta_{h} \mathrm{D}_{h}(X)$, and called later on $h$-boundary of $X$.

As discussed in various previous works (see for instance [2] for a survey), the idea of multigrid convergence is that when we define a quantity estimator on $\mathrm{D}_{h}(X)$, we check if the estimated quantity converges (theoretically and/or experimentally) to the associated one on $X$ when $h$ tends to zero. More formally,

Definition 1 (Multigrid convergence for local geometric quantities). A local discrete geometric estimator $\hat{E}$ of some geometric quantity $E$ is multigrid convergent for the family $\mathbb{X}$ if and only if, for any $X \in \mathbb{X}$, there exists a grid step $h_{X}>0$ such that the estimate $\hat{E}\left(\mathrm{D}_{h}(X), \hat{x}, h\right)$ is defined for all $\hat{x} \in \partial_{h} X$ with $0<h<h_{X}$, and for any $x \in \partial X$,

$$
\forall \hat{x} \in \partial_{h} X \text { with }\|\hat{x}-x\|_{\infty} \leq h,\left|\hat{E}\left(\mathrm{D}_{h}(X), \hat{x}, h\right)-E(X, x)\right| \leq \tau_{X, x}(h),
$$

where $\tau_{X, x}: \mathbb{R}^{+} \backslash\{0\} \rightarrow \mathbb{R}^{+}$has null limit at 0 . This function defines the speed of convergence of $\hat{E}$ toward $E$ at point $x$ of $X$. The convergence is uniform for $X$ when every $\tau_{X, x}$ is bounded from above by a function $\tau_{X}$ independent of $x \in \partial X$ with null limit at 0 . 
When a geometrical quantity is global (e.g. area or volume), we do not need explicit mapping between $\partial X$ and $\partial_{h} X$, and Definition 1 can be rephrased to define multigrid convergence of global geometric quantities [2]. A local discrete estimator thus estimates a geometric quantity at points on the $h$-frontier of a digital set, otherwise said at any point on the interpixel representation of the digital set boundary. This definition encompasses usual definitions where input points are pointels, linels or surfels.

In some proofs, a more precise mapping between points $x \in \partial X$ and $\hat{x} \in \partial_{h} X$ is required. For any shape $X \in \mathbb{R}^{d}$, the medial axis $\mathrm{MA}(\partial X)$ of $\partial X$ is the subset of $\mathbb{R}^{d}$ whose points have more than one closest point to $\partial X$. The reach reach $(X)$ of $X$ is the infimum of the distance between $\partial X$ and its medial axis. Shapes with positive reach have principal curvatures bounded by $\pm 1 / \operatorname{reach}(X)$. The projection $\pi^{X}$ is the mapping from $X \backslash \operatorname{MA}(\partial X)$ onto $\partial X$ that associates to each point its closest point in $\partial X$ (cf. Fig. 1-(right)).

This projection can be restricted to domain $\partial_{h} X$ in order to define a mapping $\pi_{h}^{X}$ from the $h$-frontier $\partial_{h} X$ to the boundary $\partial X$. This mapping was called back-projection in [33]. For any 2D shape $X$ with positive reach, for $0<h \leq \operatorname{reach}(X)$, Lemma B.9 [33] indicates that the map $\pi_{h}^{X}$ is well-defined and onto. It shows that the Hausdorff distance of boundaries $\partial_{h} X$ and $\partial X$ is no greater than $\frac{\sqrt{2}}{2} h$, hence they get closer and closer as the grid step is refined.

In $d \mathrm{D}$, it is possible to show ${ }^{2}$ that their Hausdorff distance is no greater than $\frac{\sqrt{d}}{2} h$. Furthermore, it is a known fact that $\pi^{X}$ is continuous over $\mathbb{R}^{d} \backslash \mathrm{MA}(\partial X)$, hence over $\partial_{h} X$ with an adequate $h$.

\subsection{Integral invariants theory}

In Geometry Processing, integral invariants have been widely investigated to define estimators of differential quantities (see $[14,15]$ for a complete overview). For short, the main idea is to move a kernel on points $x \in \partial X$ and to compute integrals on the intersection between $X$ and the kernel. Even if different kernels (e.g., Euclidean ball, Euclidean sphere) and different integration functions can be considered, we focus here on volumetric integral invariants defined as follows:

Definition 2. Given $X \in \mathbb{X}$ and a radius $r \in \mathbb{R}^{+*}$, the volumetric integral $V_{R}(x)$ at $x \in \partial X$ is given by (see Fig. 1-(left))

$$
V_{R}(x) \stackrel{\text { def }}{=} \int_{B_{R}(x)} \chi(p) d p,
$$

where $B_{R}(x)$ is the Euclidean ball with radius $R$ and center $x$ and $\chi(p)$ the characteristic function of $X$. In dimension 2, we simply denote $A_{R}(x)$ such quantity.

Several authors have detailed connections between $V_{R}(x)$ and curvature (resp. mean curvature) at $x$ for shapes in $\mathbb{R}^{2}\left(\right.$ resp. $\left.\mathbb{R}^{3}\right)[34,14,15]$.

\footnotetext{
${ }^{2}$ The proof follows the same lines as Lemma B.9 [33].
} 


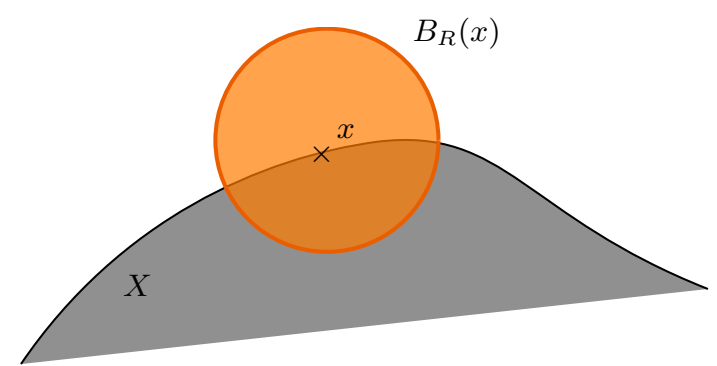

(a)

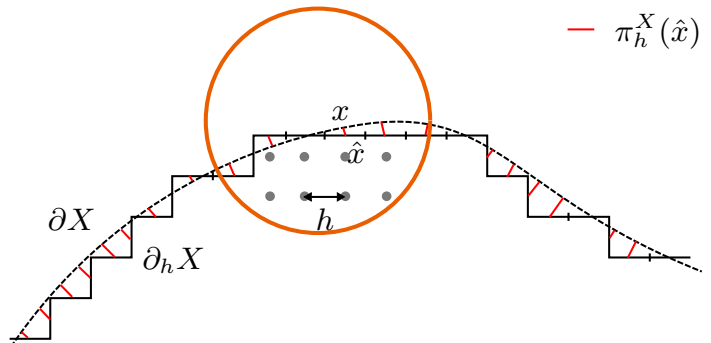

(b)

Figure 1: Integral invariant computation (left) and notations (right) in dimension 2.

Lemma 1 ([15]). For a sufficiently smooth shape $X$ in $\mathbb{R}^{2}, x \in \partial X$, we have

$$
A_{R}(x)=\frac{\pi}{2} R^{2}-\frac{\kappa(X, x)}{3} R^{3}+O\left(R^{4}\right)
$$

where $\kappa(X, x)$ is the curvature of $\partial X$ at $x$. For a sufficiently smooth shape $X$ in $\mathbb{R}^{3}$ and $x \in \partial X$, we have

$$
V_{R}(x)=\frac{2 \pi}{3} R^{3}-\frac{\pi H(X, x)}{4} R^{4}+O\left(R^{5}\right)
$$

where $H(X, x)$ is the mean curvature of $\partial X$ at $x$.

Such results are obtained by Taylor expansion at $x$ of the surface $\partial X$ approximated by a parametric function $y=f(x)$ in $2 \mathrm{D}$ and $z=f(x, y)$ in 3D. From Eq. (4) and (5) and with a fixed radius $R$, one can derive local estimators $\tilde{\kappa}_{R}$ and $\tilde{H}_{R}$ respectively:

$$
\tilde{\kappa}_{R}(X, x) \stackrel{\text { def }}{=} \frac{3 \pi}{2 R}-\frac{3 A_{R}(x)}{R^{3}}, \quad \tilde{H}_{R}(X, x) \stackrel{\text { def }}{=} \frac{8}{3 R}-\frac{4 V_{R}(x)}{\pi R^{4}}
$$

In this way, when $R$ tends to zero, both estimated values will converge to expected ones (respectively $\kappa$ and $H$ ). More formally:

$$
\tilde{\kappa}_{R}(X, x)=\kappa(X, x)+O(R), \quad \tilde{H}_{R}(X, x)=H(X, x)+O(R)
$$

Similarly, directional information such as principal curvatures and thus Gaussian curvature can be retrieved from integral computations. Indeed, instead of computing the measure of $B_{R}(x) \cap X$ as in Def. 2, we consider its covariance matrix. Given a non-empty subset $Y \subset \mathbb{R}^{d}$, the covariance matrix of $Y$ is given by

$$
J(Y) \stackrel{\text { def }}{=} \int_{Y}(p-\bar{Y})(p-\bar{Y})^{T} d p=\int_{Y} p p^{T} d p-\operatorname{Vol}(Y) \overline{Y Y}^{T},
$$

where $\bar{Y}$ is the centroid of $Y$ and $\operatorname{Vol}(Y)$ its volume. For non negative integers $p, q$ and $s$, we recall the definition of $(p, q, s)$-moments $m_{p, q, s}(Y)$ of $Y$ :

$$
m_{p, q, s}(Y) \stackrel{\text { def }}{=} \iiint_{Y} x^{p} y^{q} z^{s} d x d y d z .
$$


Note that the volume $\operatorname{Vol}(Y)$ is the 0 -moment $m_{0,0,0}(Y)$, and that the centroid $\bar{Y}$ is the vector of 1 moments normalized by the 0-moment, i.e. $\left(m_{1,0,0}(Y), m_{0,1,0}(Y), m_{0,0,1}(Y)\right)^{T} / m_{0,0,0}(Y)$. For simplicity, let us denote by $A$ the Euclidean set $B_{R}(x) \cap X$. The covariance matrix of $A$ is then rewritten $\operatorname{as}^{3}$ :

$$
J(A)=\left[\begin{array}{ccc}
m_{2,0,0}(A) & m_{1,1,0}(A) & m_{1,0,1}(A) \\
m_{1,1,0}(A) & m_{0,2,0}(A) & m_{0,1,1}(A) \\
m_{1,0,1}(A) & m_{0,1,1}(A) & m_{0,0,2}(A)
\end{array}\right]-\frac{1}{m_{0,0,0}(A)}\left[\begin{array}{c}
m_{1,0,0}(A) \\
m_{0,1,0}(A) \\
m_{0,0,1}(A)
\end{array}\right] \otimes\left[\begin{array}{c}
m_{1,0,0}(A) \\
m_{0,1,0}(A) \\
m_{0,0,1}(A)
\end{array}\right]^{T}
$$

In [14], authors have demonstrated that eigenvalues and eigenvectors of $J(A)$ provide principal curvature and principal direction information:

Lemma 2 ([14], Theorem 2). Given a shape $X \in \mathbb{X}$, the eigenvalues $\lambda_{1}, \lambda_{2}, \lambda_{3}$ of $J(A)$, where $A=$ $B_{R}(x) \cap X$ and $x \in \partial X$, have the following Taylor expansion:

$$
\begin{aligned}
& \lambda_{1}=\frac{2 \pi}{15} R^{5}-\frac{\pi}{48}\left(3 \kappa^{1}(X, x)+\kappa^{2}(X, x)\right) R^{6}+O\left(R^{7}\right) \\
& \lambda_{2}=\frac{2 \pi}{15} R^{5}-\frac{\pi}{48}\left(\kappa^{1}(X, x)+3 \kappa^{2}(X, x)\right) R^{6}+O\left(R^{7}\right) \\
& \lambda_{3}=\frac{19 \pi}{480} R^{5}-\frac{9 \pi}{512}\left(\kappa^{1}(X, x)+\kappa^{2}(X, x)\right) R^{6}+O\left(R^{7}\right)
\end{aligned}
$$

where $\kappa^{1}(X, x)$ and $\kappa^{2}(X, x)$ denotes the principal curvatures of $\partial X$ at $x .^{4}$

Hence, similarly to Eq. (6), one can define local estimators $\tilde{\kappa}_{R}^{1}, \tilde{\kappa}_{R}^{2}$ and finally the Gaussian curvature $\tilde{K}_{R} \stackrel{\text { def }}{=} \tilde{\kappa}_{R}^{1} \cdot \tilde{\kappa}_{R}^{2}$ as functions of $\left\{\lambda_{i}\right\}_{1,2,3}$ and $R$. From Lemma 2, all these estimators converge in the continuous setting when $R$ tends to 0 .

When dealing with digital shapes $\mathrm{D}_{h}(X)$, implementation of these estimators becomes straightforward: choose a radius $R$, center a Euclidean (or digital) ball at chosen points of $\partial_{h} X$ (e.g. centroids of linels or surfels), compute the quantities (area, volume, covariance matrix) and finally estimate curvature information $\tilde{\kappa}, \tilde{H}, \tilde{\kappa}^{1}, \tilde{\kappa}^{2}$ or $\tilde{K}$. However, several issues are hidden in this approach: What are meaningful values for $R$ according to the shape size and geometry? Do points of $\partial_{h} X$ converge to points $x \in \partial X$ for which Lemmas 1 and 2 are valid ? Does counting the number of pixels (resp. voxels) converge to $A_{R}(x)\left(\right.$ resp. $\left.V_{R}(x)\right)$ ? Does the digital covariance matrix converges to the expected one? The rest of the paper addresses all these questions.

\subsection{Multigrid convergence of $2 D$ and mean curvature estimator in digital space}

In [31], we have demonstrated that digital versions of estimators defined in Eq. (6) lead to efficient and multigrid convergent estimators for digitizations of smooth $2 \mathrm{D}$ shapes. In this section, we briefly describe

\footnotetext{
${ }^{3} \otimes$ denotes the usual tensor product in vector spaces.

${ }^{4}$ There is a typographic error in $\lambda_{1}$ in the paper [14].
} 
the overall structure of this proof since similar arguments will be used in Sect. 3 to demonstrate that our digital principal curvature estimators do converge uniformly.

First, we used existing results on digital area or volume estimation by counting grid points. Hence, for $2 \mathrm{D}$ shapes $X \in \mathbb{X}$ and $3 \mathrm{D}$ shapes $X^{\prime} \in \mathbb{X}$, we have

$$
\begin{aligned}
& \widehat{\operatorname{Area}}\left(\mathrm{D}_{h}(X), h\right) \stackrel{\text { def }}{=} h^{2} \operatorname{Card}\left(\mathrm{D}_{h}(X)\right)=\operatorname{Area}(X)+O\left(h^{\beta}\right), \\
& \widehat{\operatorname{Vol}}\left(\mathrm{D}_{h}\left(X^{\prime}\right), h\right) \stackrel{\text { def }}{=} h^{3} \operatorname{Card}\left(\mathrm{D}_{h}\left(X^{\prime}\right)\right)=\operatorname{Vol}\left(X^{\prime}\right)+O\left(h^{\gamma}\right),
\end{aligned}
$$

for $\beta=\gamma=1$ in the general case and $\beta=\gamma>1$ with further constraints on $\mathbb{X}$ (e.g. $C^{3}$ with non-zero curvature) $[35,36,37]$.

Then, we focused on the convergence of the area estimation on Euclidean shapes defined by $B_{R}(x) \cap X$ at $x \in \partial X$ in dimension 2. We defined a digital curvature estimator $\hat{\kappa}_{R}\left(\mathrm{D}_{h}(X), x, h\right)$ by applying the area estimation by counting on $B_{R}(x) \cap X$ and Eq. (6), see [31], Eq. (11). We first demonstrated that $\hat{\kappa}_{R}\left(\mathrm{D}_{h}(X), x, h\right)$ converges to $\kappa(X, x)$ (note that curvatures are evaluated at the same point $\left.x \in \partial X\right)$ :

Theorem 1 (Convergence of $\hat{\kappa}_{R}$ along $\partial X$, Theorem 1 of [31]). Let $X$ be some convex shape of $\mathbb{R}^{2}$, with at least $C^{2}$-boundary and bounded curvature. Then there exists positive constants $h_{0}, K_{1}$ and $K_{2}$ such that

$$
\forall h<h_{0}, R=k_{m} h^{\alpha_{m}}, \forall x \in \partial X,\left|\hat{\kappa}_{R}\left(\mathrm{D}_{h}(X), x, h\right)-\kappa(X, x)\right| \leq K h^{\alpha_{m}},
$$

where $\alpha_{m}=\frac{\beta}{2+\beta}, k_{m}=\left((1+\beta) K_{1} / K_{2}\right)^{\frac{1}{2+\beta}}, K=K_{2} k_{m}+3 K_{1} / k_{m}^{1+\beta}$. In the general case, $\alpha_{m}=\frac{1}{3}$.

Then, we showed that moving the digital estimation from $x \in \partial X$ to $\hat{x} \in \partial_{h} X$ does not change the convergence results:

Theorem 2 (Uniform convergence $\hat{\kappa}_{R}$ along $\partial_{h} X$, Theorem 2 of [31]). Let $X$ be some convex shape of $\mathbb{R}^{2}$, with at least $C^{3}$-boundary and bounded curvature. Then, there exists positive constants $h_{0}$ and $k$, for any $h \leq h_{0}$, setting $r=k h^{\frac{1}{3}}$, we have

$$
\forall x \in \partial X, \forall \hat{x} \in \partial_{h} X,\|\hat{x}-x\|_{\infty} \leq h \Rightarrow\left|\hat{\kappa}_{R}\left(\mathrm{D}_{h}(X), \hat{x}, h\right)-\kappa(X, x)\right| \leq K h^{\frac{1}{3}}
$$

In [31], we also presented similar results and convergence speed for mean curvature estimation in 3D from digital volume estimation.

To demonstrate that principal curvature estimators can be defined from digital version of integral invariants, we use the exact same process:

1. We first demonstrate that digital estimations of covariance matrix are multigrid convergent (Sect. 3.1 and 3.2);

2. Then, we give explicit error bounds on both the geometrical moments and the covariance matrix when we change the reference point from $x \in \partial X$ to $\hat{x} \in \partial_{h} X$ (Sect. 3.3 and 3.4); 
3. Finally, we gather all these results to demonstrate that principal curvature estimators are uniformly multigrid convergent for all $\hat{x} \in \partial_{h} X$ (Sect. 3.5).

\section{Multigrid convergence of principal curvature estimators in digital space}

In this section, we derive digital principal curvature and principal direction estimators by digital approximation of local covariance matrices. Convergence results rely on the fact that digital moments converge in the same manner as volumes [37]. In the whole section, the considered family of shapes $\mathbb{X}$ is composed of compact subsets of $\mathbb{R}^{3}$ with positive reach, the boundary of which is $C^{3}$ and can be decomposed in a finite number of monotonous pieces. Compacity is required so that the boundary belongs to the shape. $C^{3}$ smoothness is required in the limited developments of Pottmann et al. [14, 15] relating covariance matrix and curvatures. Positive reach guarantees that two pieces of boundaries are not too close to each other, and this fact is also required in the previous limited developments (although this is not stated in their paper). The finite decomposition into monotonous pieces induces that integrals as limit of sums converge at speed at least $O(h)$.

\subsection{Convergence of digital moments}

Following the same principles as the area and volume estimators by counting, we define the digital $(p, q, s)$-moments $\hat{m}_{p, q, s}(Z, h)$ of a subset $Z$ of $\mathbb{Z}^{3}$ at step $h$ as

$$
\hat{m}_{p, q, s}(Z, h) \stackrel{\text { def }}{=} h^{3+p+q+s} M_{p, q, s}(Z)
$$

where $M_{p, q, s}(Z) \stackrel{\text { def }}{=} \sum_{(i, j, k) \in Z} i^{p} j^{q} k^{s}$. To shorten expressions, we denote by $\sigma$ the sum $p+q+s$, which will always be an integer in $\{0,1,2\}$.

There exist multigrid convergent results for digital moments that are similar to the multigrid convergence of the area and the volume estimator (see Eq.(14)). Since their speed of convergence depends on the order $\sigma$ of the moment, we may thus write for some constant $\mu_{\sigma} \geq 1[37]$ :

$$
\hat{m}_{p, q, s}\left(\mathrm{D}_{h}(Y), h\right)=m_{p, q, s}(Y)+O\left(h^{\mu_{\sigma}}\right) .
$$

The involved constants $\mu_{i}$ are at least 1 in the general case, and some authors have established better bounds in places where the Gaussian curvature does not vanish (e.g. see [38] where $\mu_{0}=\frac{38}{25}-\epsilon$, or [39], Theorem 1, where $\left.\mu_{0}=\frac{66}{43}-\epsilon\right)$.

We wish to apply this formula to the set $A=B_{R}(x) \cap X$, whose size decreases with $h$. Big "O" notation in Eq. (17) hides the fact that the involved constant depends on the shape size, scale and maximal curvature. Hence, we need to normalize our moment estimation so that the error is no more influenced by the scale: 


$$
\begin{aligned}
\hat{m}_{p, q, s}\left(\mathrm{D}_{h}(A), h\right) & =h^{3+\sigma} M_{p, q, s}\left(\left(\frac{1}{h} \cdot B_{R}(x) \cap X\right) \cap \mathbb{Z}^{3}\right) \\
& =h^{3+\sigma} M_{p, q, s}\left(\frac{R}{h} \cdot\left(B_{1}\left(\frac{1}{R} \cdot x\right) \cap \frac{1}{R} \cdot X\right) \cap \mathbb{Z}^{3}\right) \\
& =R^{3+\sigma}\left(\frac{h}{R}\right)^{3+\sigma} M_{p, q, s}\left(\mathrm{D}_{h / R}\left(B_{1}\left(\frac{1}{R} \cdot x\right) \cap \frac{1}{R} \cdot X\right)\right) \\
& =R^{3+\sigma} \hat{m}_{p, q, s}\left(\mathrm{D}_{h / R}\left(B_{1}\left(\frac{1}{R} \cdot x\right) \cap \frac{1}{R} \cdot X\right), \frac{h}{R}\right) .
\end{aligned}
$$

The shape $B_{1}\left(\frac{1}{R} \cdot x\right) \cap \frac{1}{R} \cdot X$ tends toward a half-ball of radius 1 as $R$ decreases. Therefore, we may apply Eq.(17) on Eq.(18) and consider that the involved constant does not depend on $R$ or $h$. Note that we use below the obvious relation $m_{p, q, s}(R \cdot Y)=R^{3+\sigma} m_{p, q, s}(Y)$.

$$
\begin{aligned}
\hat{m}_{p, q, s}\left(\mathrm{D}_{h}(A), h\right) & =R^{3+\sigma}\left(m_{p, q, s}\left(B_{1}\left(\frac{1}{R} \cdot x\right) \cap \frac{1}{R} \cdot X\right)+O\left(\frac{h}{R}\right)^{\mu_{\sigma}}\right) \\
& =m_{p, q, s}\left(B_{R}(x) \cap X\right)+O\left(R^{3+\sigma-\mu_{\sigma}} h^{\mu_{\sigma}}\right) \\
& =m_{p, q, s}(A)+O\left(R^{3+\sigma-\mu_{\sigma}} h^{\mu_{\sigma}}\right) .
\end{aligned}
$$

Eq.(19) is a multigrid convergent result for digital moments of subsets $B_{R}(x) \cap X$ valid for $R$ decreasing as $h$ decreases.

\subsection{Digital approximation of covariance matrix around a point $x$}

For any digital subset $Z \subset \mathbb{Z}^{3}$, we define its digital covariance matrix $\hat{J}(Z, h)$ at step $h$ as:

$$
\begin{aligned}
& \hat{J}(Z, h) \stackrel{\text { def }}{=}\left[\begin{array}{ccc}
\hat{m}_{2,0,0}(Z, h) & \hat{m}_{1,1,0}(Z, h) & \hat{m}_{1,0,1}(Z, h) \\
\hat{m}_{1,1,0}(Z, h) & \hat{m}_{0,2,0}(Z, h) & \hat{m}_{0,1,1}(Z, h) \\
\hat{m}_{1,0,1}(Z, h) & \hat{m}_{0,1,1}(Z, h) & \hat{m}_{0,0,2}(Z, h)
\end{array}\right] \\
&-\frac{1}{\hat{m}_{0,0,0}(Z, h)}\left[\begin{array}{c}
\hat{m}_{1,0,0}(Z, h) \\
\hat{m}_{0,1,0}(Z, h) \\
\hat{m}_{0,0,1}(Z, h)
\end{array}\right] \otimes\left[\begin{array}{c}
\hat{m}_{1,0,0}(Z, h) \\
\hat{m}_{0,1,0}(Z, h) \\
\hat{m}_{0,0,1}(Z, h)
\end{array}\right]^{T} .
\end{aligned}
$$

We now establish the multigrid convergence of the digital covariance matrix toward the covariance matrix. In this case, we know the exact position of the point $x$ at which both digital and continuous covariance matrices are computed. The following theorem only takes into account the integral approximation error.

Theorem 3 (Multigrid convergence of digital covariance matrix). Let $X \in \mathbb{X}$. Then, there exists some constant $h_{X}$, such that for any grid step $0<h<h_{X}$, for arbitrary $x \in \mathbb{R}^{3}$, for arbitrary $R \geq h$, with non-empty $A(R, x) \stackrel{\text { def }}{=} B_{R}(x) \cap X$, we have:

$$
\left\|\hat{J}\left(\mathrm{D}_{h}(A(R, x)), h\right)-J(A(R, x))\right\| \leq O\left(R^{5-\mu_{0}} h^{\mu_{0}}\right)+O\left(R^{5-\mu_{1}} h^{\mu_{1}}\right)+O\left(R^{5-\mu_{2}} h^{\mu_{2}}\right) .
$$


The constants hidden in the big $O$ do not depend on the shape size or geometry. $\|\cdot\|$ denotes the spectral norm on matrices.

Proof. To simplify expressions, we set $A \stackrel{\text { def }}{=} A(R, x), A_{h} \stackrel{\text { def }}{=} \mathrm{D}_{h}(A(R, x))$. We begin by translating the sets $A$ and $A_{h}$ towards the origin w.r.t. x. We must use a vector that takes into account the digitization, hence we shift $A_{h}$ by the vector $\left[\frac{x}{h}\right]$, the integer vector closest to $\frac{x}{h}$, and we shift $A$ with the vector $h\left[\frac{x}{h}\right]$. We further set $\tilde{A}_{h} \stackrel{\text { def }}{=} \mathrm{D}_{h}(A)-\left[\frac{x}{h}\right]$ and $\tilde{A} \stackrel{\text { def }}{=} A-h\left[\frac{x}{h}\right]$. Following these definitions,

$$
\hat{J}\left(\mathrm{D}_{h}(A(R, x)), h\right)=\hat{J}\left(A_{h}, h\right)=\hat{J}\left(\tilde{A}_{h}+\left[\frac{x}{h}\right], h\right) .
$$

Using Lemma 4 (see Appendix A), we have

$$
\hat{J}\left(\tilde{A}_{h}+\left[\frac{x}{h}\right], h\right)=\hat{J}\left(\tilde{A}_{h}, h\right) .
$$

Writing down the definition of digital covariance matrix (see Eq.(20)), we have:

$$
\begin{aligned}
\hat{J}\left(\tilde{A}_{h}, h\right)= & {\left[\begin{array}{cc}
\hat{m}_{2,0,0}\left(\tilde{A}_{h}, h\right) & \\
& \ddots
\end{array}\right] } \\
& -\frac{1}{\hat{m}_{0,0,0}\left(\tilde{A}_{h}, h\right)}\left[\begin{array}{c}
\hat{m}_{1,0,0}\left(\tilde{A}_{h}, h\right) \\
\vdots
\end{array}\right] \otimes\left[\begin{array}{c}
\hat{m}_{1,0,0}\left(\tilde{A}_{h}, h\right) \\
\vdots
\end{array}\right]^{T} .
\end{aligned}
$$

We remark that $\tilde{A}_{h}=\mathrm{D}_{h}(A)-\left[\frac{x}{h}\right]=\mathrm{D}_{h}\left(A-h\left[\frac{x}{h}\right]\right)=\mathrm{D}_{h}(\tilde{A})$. Consequently, we apply convergence result of Eq.(19) onto set $\tilde{A}$ and insert them into Eq.(23) to get

$$
\begin{aligned}
\hat{J}\left(\tilde{A}_{h}, h\right) & =\left[\begin{array}{cc}
m_{2,0,0}(\tilde{A})+O\left(R^{5-\mu_{2}} h^{\mu_{2}}\right) & \\
& \ddots
\end{array}\right] \\
& -\frac{1}{m_{0,0,0}(\tilde{A})+O\left(R^{3-\mu_{0}} h^{\mu_{0}}\right)}\left[\begin{array}{cc}
\left(m_{1,0,0}(\tilde{A})+O\left(R^{4-\mu_{1}} h^{\mu_{1}}\right)\right)^{2} & \\
& \ddots
\end{array}\right] .
\end{aligned}
$$

Note that constants in big $\mathrm{O}$ are independent of $X$ thanks to the normalization. In Eq.(24), we recognize easily $J(\tilde{A})$ plus other terms. We upper bound the other terms with two facts: (i) the radius $R$ is greater than $h$, (ii) since $\tilde{A}$ is non-empty and close to the origin, we apply Eq.(A.2) and Eq.(A.4) of Lemma 5 for set $\tilde{A} \subset B_{R}(t)$ with $t=x-h\left[\frac{x}{h}\right]$, noticing that $\|t\|_{\infty} \leq \frac{h}{2}$. We obtain

$$
\hat{J}\left(\tilde{A}_{h}, h\right)=J(\tilde{A})+O\left(R^{5-\mu_{2}} h^{\mu_{2}}\right)+O\left(R^{5-\mu_{0}} h^{\mu_{0}}\right)+O\left(R^{5-\mu_{1}} h^{\mu_{1}}\right) .
$$

We conclude since $J(\tilde{A})=J\left(A-h\left[\frac{x}{h}\right]\right)=J(A)$ by Lemma 4 (see Appendix A).

\subsection{Influence of a positioning error on moments}

We do not know generally the exact position of $x$ but only some approximation $\hat{x}$ taken on the digital boundary $\partial_{h} X$. We therefore examine the perturbation of the moments when they are evaluated at a shifted position $x+\mathbf{t}$. 
Lemma 3. For any subset $X \subset \mathbb{R}^{3}$ and any vector $\mathbf{t}$ with norm $t \stackrel{\text { def }}{=}\|\mathbf{t}\|_{2} \leq R$, we have for $0 \leq p+q+s \stackrel{\text { def }}{=}$ $\sigma \leq 2$ :

$$
m_{p, q, s}\left(B_{R}(x+\mathbf{t}) \cap X\right)=m_{p, q, s}\left(B_{R}(x) \cap X\right)+\sum_{i=0}^{\sigma} O\left(\|x\|^{i} t R^{2+\sigma-i}\right) .
$$

The proof is detailed in Appendix A.2.

\subsection{Influence of a positioning error on covariance matrix}

We now establish the multigrid convergence of the digital covariance matrix toward the covariance matrix even when the exact point $x$ is unknown.

Theorem 4. (Multigrid convergence of digital covariance matrix with position error.) Let $X \in$ $\mathbb{X}$. Then, there exists some constant $h_{X}$, such that for any grid step $0<h<h_{X}$, for arbitrary $R \geq h$, for any $x \in \partial X$ and any $\hat{x} \in \partial_{h} X,\|x-\hat{x}\|_{\infty} \leq h$, we have:

$$
\left\|\hat{J}\left(\mathrm{D}_{h}(A(R, \hat{x})), h\right)-J(A(R, x))\right\| \leq O\left(\|x-\hat{x}\| R^{4}\right)+\sum_{i=0}^{2} O\left(R^{5-\mu_{i}} h^{\mu_{i}}\right),
$$

with $A(R, y) \stackrel{\text { def }}{=} B_{R}(y) \cap X$. The constants hidden in the big $O$ do not depend on the shape size or geometry. Proof. The fact that $\|x-\hat{x}\|_{\infty} \leq h \leq R$ induces that $A(R, x)$ and $A(R, \hat{x})$ are both non-empty. We cut the difference of two matrices into two parts:

$$
\begin{aligned}
\left\|\hat{J}\left(\mathrm{D}_{h}(A(R, \hat{x})), h\right)-J(A(R, x))\right\| \leq & \left\|\hat{J}\left(\mathrm{D}_{h}(A(R, \hat{x})), h\right)-J(A(R, \hat{x}))\right\| \\
& +\|J(A(R, \hat{x}))-J(A(R, x))\| .
\end{aligned}
$$

For the first error term, we apply directly Theorem 3 at point $\hat{x}$. For the second term, we set $\mathbf{t} \stackrel{\text { def }}{=} \hat{x}-x$, $t \stackrel{\text { def }}{=}\|\mathbf{t}\|$. Then we use the invariance of the covariance matrix with respect to translation to shift the problem toward the origin:

$$
\begin{aligned}
\|J(A(R, \hat{x}))-J(A(R, x))\| & =\|J(A(R, x+\mathbf{t}))-J(A(R, x))\| \\
& =\|J(A(R, x+\mathbf{t})-x)-J(A(R, x)-x)\| \\
& =\left\|J\left(\left(B_{R}(x+\mathbf{t})-x\right) \cap(X-x)\right)-J\left(\left(B_{R}(x)-x\right) \cap(X-x)\right)\right\| \\
& =\left\|J\left(B_{R}(\mathbf{t}) \cap(X-x)\right)-J\left(B_{R}(0) \cap(X-x)\right)\right\| \\
& =\left\|J\left(B_{R}(\mathbf{t}) \cap X^{\prime}\right)-J\left(B_{R}(0) \cap X^{\prime}\right)\right\|,
\end{aligned}
$$


with $X^{\prime} \stackrel{\text { def }}{=} X-x$. We will apply Lemma 3 for the different moments in the covariance matrix $J$. We denote by $Y_{t}$ the set $B_{R}(\mathbf{t}) \cap X^{\prime}$ and by $Y_{0}$ the set $B_{R}(0) \cap X^{\prime}$.

$$
\begin{aligned}
\left\|J\left(Y_{t}\right)-J\left(Y_{0}\right)\right\|= & \|\left[\begin{array}{cc}
m_{2,0,0}\left(Y_{t}\right)-m_{2,0,0}\left(Y_{0}\right) & \\
& \ddots
\end{array}\right] \\
& -\frac{1}{m_{0,0,0}\left(Y_{t}\right)}\left[\begin{array}{c}
m_{1,0,0}\left(Y_{t}\right) \\
\vdots
\end{array}\right] \otimes\left[\begin{array}{c}
m_{1,0,0}\left(Y_{t}\right) \\
\vdots
\end{array}\right]^{T} \\
& +\frac{1}{m_{0,0,0}\left(Y_{0}\right)}\left[\begin{array}{c}
m_{1,0,0}\left(Y_{0}\right) \\
\vdots
\end{array}\right] \otimes\left[\begin{array}{c}
m_{1,0,0}\left(Y_{0}\right) \\
\vdots
\end{array}\right]^{T} \| .
\end{aligned}
$$

Matrix $J\left(Y_{t}\right)-J\left(Y_{0}\right)$ contains differences of geometrical moments of order two (e.g. $m_{2,0,0}\left(Y_{t}\right)-$ $\left.m_{2,0,0}\left(Y_{0}\right)\right)$ and quantities in the form of $\Delta \stackrel{\text { def }}{=} \frac{m_{1,0,0}\left(Y_{t}\right)^{2}}{m_{0,0,0}\left(Y_{t}\right)}-\frac{m_{1,0,0}\left(Y_{0}\right)^{2}}{m_{0,0,0}\left(Y_{0}\right)}\left(\operatorname{component}(1,1)\right.$ in $J\left(Y_{t}\right)-J\left(Y_{0}\right)$ matrix). From Lemma 3, every error on second-order moments is in $O\left(t R^{4}\right)$. To bound $\Delta$ quantities, we first observe that $\left|m_{0,0,0}\left(Y_{t}\right)-m_{0,0,0}\left(Y_{0}\right)\right|=\pi R^{2}\left(t+O\left(t^{2}\right)+O\left(t R^{2}\right)\right)$ using Theorem 7 in [15]. Hence,

$$
\begin{array}{rlrl}
\Delta & =\frac{m_{1,0,0}\left(Y_{t}\right)^{2}}{m_{0,0,0}\left(Y_{0}\right)+O\left(t R^{2}\right)}-\frac{m_{1,0,0}\left(Y_{0}\right)^{2}}{m_{0,0,0}\left(Y_{0}\right)} & \\
& =O\left(t R^{2}\right) \frac{m_{1,0,0}\left(Y_{t}\right)^{2}}{m_{0,0,0}\left(Y_{0}\right)^{2}}+\frac{m_{1,0,0}\left(Y_{t}\right)^{2}-m_{1,0,0}\left(Y_{0}\right)^{2}}{m_{0,0,0}\left(Y_{0}\right)} & \left(\text { since } \frac{a}{b+O(x)}=\frac{a}{b}+\frac{a}{b^{2}} O(x)\right) \\
& =O\left(t R^{4}\right)+\left(m_{1,0,0}\left(Y_{t}\right)+m_{1,0,0}\left(Y_{0}\right)\right) \frac{m_{1,0,0}\left(Y_{t}\right)-m_{1,0,0}\left(Y_{0}\right)}{m_{0,0,0}\left(Y_{0}\right)} & & \text { Lemma } \left.3 \text { and } a^{2}-b^{2}=(a-b)(a+b)\right) \\
& =O\left(t R^{4}\right)+\left(O\left(t R^{3}\right)+O\left(R^{4}\right)\right) \frac{m_{1,0,0}\left(Y_{t}\right)-m_{1,0,0}\left(Y_{0}\right)}{m_{0,0,0}\left(Y_{0}\right)} & & \text { (Lemma 5, Eq.(A.2)) } \\
& =O\left(t R^{4}\right)+\left(O\left(t R^{3}\right)+O\left(R^{4}\right)\right) \frac{O\left(t R^{3}\right)}{m_{0,0,0}\left(Y_{0}\right)} & & \text { (Lemma 3) } \\
& =O\left(t R^{4}\right) & \left.\quad \text { (since } t<R \text { and } m_{0,0,0}\left(Y_{0}\right)=O\left(R^{3}\right)\right)
\end{array}
$$

The same bound is found for all terms of the matrix. Putting everything together gives the result.

\subsection{Convergence for $\hat{x} \in \partial_{h} X$}

Following the limited development of Lemma 2, we define estimators of curvatures from the diagonalization of the digital covariance matrix.

Definition 3. Let $Z$ be a digital shape, $x$ some point of $\mathbb{R}^{3}$ and $h>0$ a gridstep. For $R \geq h$, we define the integral principal curvature estimators $\hat{\kappa}_{R}^{1}$ and $\hat{\kappa}_{R}^{2}$ of $Z$ at point $y \in \mathbb{R}^{3}$ and step $h$ as

$$
\begin{aligned}
\hat{\kappa}_{R}^{1}(Z, y, h) & =\frac{6}{\pi R^{6}}\left(\hat{\lambda}_{2}-3 \hat{\lambda}_{1}\right)+\frac{8}{5 R}, \\
\hat{\kappa}_{R}^{2}(Z, y, h) & =\frac{6}{\pi R^{6}}\left(\hat{\lambda}_{1}-3 \hat{\lambda}_{2}\right)+\frac{8}{5 R},
\end{aligned}
$$

where $\hat{\lambda}_{1}$ and $\hat{\lambda}_{2}$ are the two greatest eigenvalues of $\left.\hat{J}\left(B_{R / h}\left(\frac{1}{h} \cdot y\right) \cap Z, h\right)\right)$. 
We recall the following result of matrix perturbation theory [40, 41]:

Theorem 5 (Lidskii-Weyl inequality). If $\lambda_{i}(B)$ denotes the ordered eigenvalues of some symmetric matrix $B$ and $\lambda_{i}(B+E)$ the ordered eigenvalues of some symmetric matrix $B+E$, then $\max _{i} \mid \lambda_{i}(B)-\lambda_{i}(B+$ $E) \mid \leq\|E\|$.

We prove below that our integral principal curvature estimators are multigrid convergent toward the principal curvatures along the shape.

Theorem 6. Uniform convergence of principal curvature estimators $\hat{\kappa}_{R}^{1}$ and $\hat{\kappa}_{R}^{2}$ along $\partial_{h} X$. Let $X \in \mathbb{X}$. For $i \in\{1,2\}$, recall that $\kappa^{i}(X, x)$ is the $i$-th principal curvature of $\partial X$ at boundary point $x$. Then, there exist positive constants $h_{X}, k, K$ such that, for any $h \leq h_{X}$, setting $R=k h^{\frac{1}{3}}$, we have

$$
\forall x \in \partial X, \forall \hat{x} \in \partial_{h} X,\|\hat{x}-x\|_{\infty} \leq h \Rightarrow\left|\hat{\kappa}_{R}^{i}\left(\mathrm{D}_{h}(X), \hat{x}, h\right)-\kappa^{i}(X, x)\right| \leq K h^{\frac{1}{3}} .
$$

Proof. We prove the result for the first principal curvature, the proof for the second one is similar. According to Definition 3, $\hat{\lambda}_{1}$ and $\hat{\lambda}_{2}$ are the two greatest eigenvalues of $\hat{J}\left(B_{R / h}\left(\frac{1}{h} \cdot \hat{x}\right) \cap Z, h\right)$ with $\left.Z=\mathrm{D}_{h}(X)\right)$. We derive easily:

$$
\begin{aligned}
\hat{J}\left(B_{R / h}\left(\frac{1}{h} \cdot \hat{x}\right) \cap \mathrm{D}_{h}(X), h\right) & =\hat{J}\left(B_{R / h}\left(\frac{1}{h} \cdot \hat{x}\right) \cap\left(\frac{1}{h} \cdot X\right) \cap \mathbb{Z}^{3}, h\right) \\
& =\hat{J}\left(\frac{1}{h} \cdot\left(B_{R}(\hat{x}) \cap X\right) \cap \mathbb{Z}^{3}, h\right) \\
& =\hat{J}\left(\mathrm{D}_{h}\left(B_{R}(\hat{x}) \cap X\right), h\right) \\
& =\hat{J}\left(\mathrm{D}_{h}(A(R, \hat{x})), h\right) .
\end{aligned}
$$

Theorem 4 indicates that $\hat{J}\left(\mathrm{D}_{h}(A(R, \hat{x})), h\right)$ and $J(A(R, x))$ are close to each other with a norm difference bounded by $O\left(\|x-\hat{x}\| R^{4}\right)+\sum_{i=0}^{2} O\left(R^{5-\mu_{i}} h^{\mu_{i}}\right)$. Since both matrices are symmetric by definition, Theorem 5 implies that $\hat{\lambda}_{1}$ and $\hat{\lambda}_{2}$ are close to the eigenvalues $\lambda_{1}(J(A(R, x)))$ and $\lambda_{2}(J(A(R, x)))$ with the same bound ${ }^{5}$. We thus write:

$$
\begin{aligned}
\hat{\kappa}_{R}^{1}\left(\mathrm{D}_{h}(X), \hat{x}, h\right) & =\frac{6}{\pi R^{6}}\left(\hat{\lambda}_{2}-3 \hat{\lambda}_{1}\right)+\frac{8}{5 R} \\
& =\frac{6}{\pi R^{6}}\left(\lambda_{2}-3 \lambda_{1}+O\left(\|x-\hat{x}\| R^{4}\right)+\sum_{i=0}^{2} O\left(R^{5-\mu_{i}} h^{\mu_{i}}\right)\right)+\frac{8}{5 R} .
\end{aligned}
$$

We then substitute the limited development of Lemma 2 into the latter equation and we bound $\|x-\hat{x}\|$ by $h$. After some calculations, we get:

$$
\hat{\kappa}_{R}^{1}\left(\mathrm{D}_{h}(X), \hat{x}, h\right)=\kappa^{1}(X, x)+O(R)+O\left(h / R^{2}\right)+\sum_{i=0}^{2} O\left(h^{\mu_{i}} / R^{1+\mu_{i}}\right) .
$$

\footnotetext{
${ }^{5}$ Note that since error bounds tend to zero as $h$ tends to zero, the ordering of the eigenvalues in both matrices is the same for a sufficiently small $h$.
} 
Setting $R=k h^{\alpha}$, we optimize the value $\alpha$ to minimize all errors. Since $\mu_{i} \geq 1$ for the shape $X$, the optimal value is $\alpha=\frac{1}{3}$. The bound follows.

It is worthy to note that the preceding error bound could be improved at neighborhoods where the gaussian curvature does not vanish: the constants $\mu_{i}$ are then closer to 1.5. However, there is the issue of estimating more precisely the position of $\hat{x}$ with respect to $x$. In the best known case, uniform convergence with bound $\approx O\left(h^{0.434}\right)$ can be expected for radius $R$ with a size proportional to $h^{0.434}$.

In Theorem 6, we focused on multigrid convergence of principal curvature quantities. However, similar results can be obtained for principal curvature directions as well.

\section{Experimental evaluation}

We present an experimental evaluation of curvature estimators in 2D and 3D (mean and principal curvatures). We have implemented our Integral Invariant estimators (II) in the open-source library DGTAL [32]. DGtal allows us to construct parametric or implicit shapes in dimension 2 and 3 for any gridstep $h$. Furthermore, DGTAL allows comparison with former approaches available in dimension 2: curvature from Most-centered Maximal Segment with length information (MDSS) [42, 23], curvature from Most-centered Digital Circular Arc (MDCA) [27] and Binomial based convolution (BC) [25]; and in dimension 3: curvature from polynomial surface approximation (Jet Fitting) [16] using a binding between DGTAL and CGAL [43]. Jet Fitting approach requires a point set on which the polynomial fitting is performed. In our multigrid setting, the radius of the spherical kernel used to construct the local point-set around a given surface element is the same as the radius of the integral invariant kernel $\left(R=k_{m} h^{\alpha_{m}}\right)$.

As described in Section 2, brute-force implementation is trivial. We first need to construct a kernel from a Euclidean ball with radius given by $R=k_{m} h^{\alpha_{m}}$ as described in theorem statements. Then, the digital object boundary is tracked and the kernel is centered on each surface elements. For 2D and 3D mean curvature estimators, the volumetric integral of the intersection between the kernel and the object is computed; for 3D principal curvature estimators, the covariance matrix of this intersection is computed and then eigenvalues and eigenvectors are deduced from it by diagonalization.

With this approach, we achieve a computational cost of $O\left((R / h)^{d}\right)$ per surface element (i.e. the size of the kernel at grid-step $h$ ). However, we can take advantage of the digital surface structure to considerably speed up this algorithm: if we consider a surface tracker for which surface elements are processed by proximity (the current surface element is a neighbor of the previous one through a translation vector $\vec{\delta}$ ), the area/volume estimation can be done incrementally. Indeed, they are countable additive:

$$
\begin{aligned}
& \widehat{\text { Area }}\left(\mathrm{D}_{h}(X) \cap B_{R}(x+\vec{\delta}), h\right)=\widehat{\operatorname{Area}}\left(\mathrm{D}_{h}(X) \cap B_{R}(x), h\right) \\
& +\widehat{\operatorname{Area}}\left(\mathrm{D}_{h}(X) \cap\left(B_{R}(x+\vec{\delta}) \backslash B_{R}(x)\right), h\right)-\widehat{\operatorname{Area}}\left(\mathrm{D}_{h}(X) \cap\left(B_{R}(x) \backslash B_{R}(x+\vec{\delta})\right), h\right) .
\end{aligned}
$$




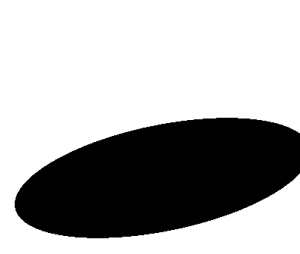

(a)

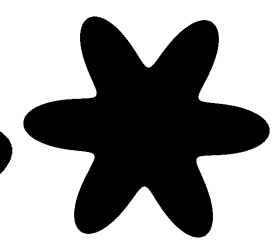

(b)

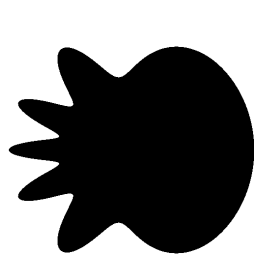

(c)

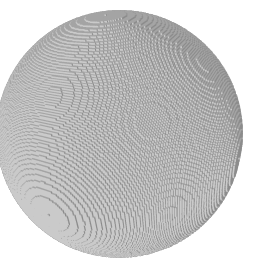

(d)

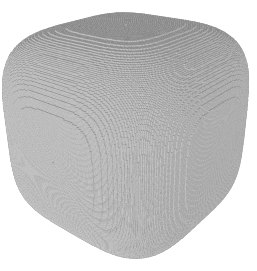

(e)

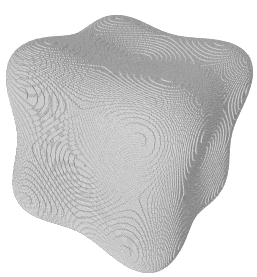

(f)

Figure 2: Illustrations of 2D and 3D shapes considered in the experimental evaluation (please refer to Table 1 for equations and parameters): Ellipse (a), Flower $(b)$, Accelerated Flower $(c)$, Sphere $(d)$, Rounded cube $(e)$ and Goursat's surface $(f)$.

Similarly we have for moments:

$$
\begin{aligned}
& \hat{m}_{p, q, s}\left(\mathrm{D}_{h}(X) \cap B_{R}(x+\vec{\delta}), h\right)=\hat{m}_{p, q, s}\left(\mathrm{D}_{h}(X) \cap B_{R}(x), h\right) \\
& \quad+\hat{m}_{p, q, s}\left(\mathrm{D}_{h}(X) \cap\left(B_{R}(x+\vec{\delta}) \backslash B_{R}(x)\right), h\right)-\hat{m}_{p, q, s}\left(\mathrm{D}_{h}(X) \cap\left(B_{R}(x) \backslash B_{R}(x+\vec{\delta})\right), h\right) .
\end{aligned}
$$

Then, if we precompute all kernels $\mathrm{D}_{h}\left(B_{R}(0 \pm \vec{\delta}) \backslash B_{R}(0)\right)$ for some $\vec{\delta}$ displacements (based on surface element umbrella configurations, 8 in $2 \mathrm{D}$ and 26 in $3 \mathrm{D}$ for $\|\vec{\delta}\|_{\infty}=h$ ), the computational cost per surface element can be reduced to $O\left((R / h)^{d-1}\right)$. Finally, in the ideal case of a Hamiltonian traversal of the surface, only the first surfel has to be computed using kernel $B_{R}(\hat{x})$ and every subsequent neighboring surfel is processed using sub-kernels $\mathrm{D}_{h}\left(B_{R}(0 \pm \vec{\delta}) \backslash B_{R}(0)\right)$.

In our experimental evaluation, we need to compare the estimated curvature values with expected Euclidean ones on parametric curves and surfaces on which such curvature information is known. We have chosen three 2D shapes to perform our evaluation : an ellipse (Fig. 2- $(a)$ ) which matches theorem hypotheses (convex $C^{3}$ shape), a flower (Fig. 2-(b)) and an accelerated flower (Fig. 2-(c)) which do not satisfy exactly theorem hypotheses $\left(C^{3}\right.$ but non-convex shapes). In 3D, we chose a sphere (Fig. 2- $(d)$ ), a rounded cube (Fig. 2-(e)) and Goursat's surface (Fig. 2-(f)). As in 2D, the sphere and the rounded cube match theorem hypotheses, and we have a non-convex shape with the Goursat's surface. Equations, parameters and shape domains of these Euclidean objects are given in Table 1. In order to quantitatively interpret error measurements given in the graphs, we detail expected minimum and maximal curvature values in this table. To compensate subpixel/subvoxel digitization effects when digitizing a continuous object, we evaluate the estimators on digitization of 10 random translations of the continuous objects (continuous objects are translated by a vector randomly selected in $[-1,1]^{2}$ or $\left.[-1,1]^{3}\right)$. Estimated quantities are compared to expected Euclidean ones for each pair of digital/continuous points contour $\left(\hat{x} \in \partial_{h} X\right.$ and $\left.x \in \partial X\right)$. In our experiments, we consider two different metrics on point-wise errors to get a global error measurement. First $l_{\infty}$ metric is used to quantify worst-case error since it reflects the uniform convergence of Theorem 2 and Theorem 6. In other experiments, we also consider $l_{2}$ error to get an average error analysis.

From Theorem 2 and Theorem 6, theory indicates that the best candidate for $\alpha_{m}$ is $\frac{1}{3}$ to ensure a multigrid 


\begin{tabular}{|l|l|c|c|c|c|}
\hline Shape & $\begin{array}{l}\text { Equation }(\text { parametric in } 2 \mathrm{D}, \text { implicit in } \\
\text { 3D) }\end{array}$ & Parameters & Domain & $k_{\text {min }}$ & $k_{\text {max }}$ \\
\hline \hline Ellipse & $\begin{array}{l}(x(t), y(t))=(\rho(t) \cdot \cos (t), \rho(t) \cdot \sin (t)) \\
\text { with } \rho(t)=\frac{b}{\sqrt{1-\left(a^{2}-b^{2}\right) / a^{2} \cdot \cos (t+\phi)}}\end{array}$ & $(a, b)=(20,7)$ & {$[-20,20]^{2}$} & 0.0175 & 0.408 \\
\hline Flower & $\begin{array}{l}(x(t), y(t))=(\rho(t) \cdot \cos (t), \rho(t) \cdot \sin (t)) \\
\text { with } \rho(t)=r_{1}+r_{2} \cdot \cos (p \cdot t)\end{array}$ & $\left(r_{1}, r_{2}, p\right)=(20,7,6)$ & {$[-20,20]^{2}$} & -1.4142 & 0.3827 \\
\hline AccFlower & $\begin{array}{l}(x(t), y(t))=(\rho(t) \cdot \cos (t), \rho(t) \cdot \sin (t)) \\
\text { with } \rho(t)=r_{1}+r_{2} \cdot \cos \left(p \cdot t^{3}\right)\end{array}$ & $\left(r_{1}, r_{2}, p\right)=(20,5,3)$ & {$[-20,20]^{2}$} & -10.4475 & 3.14815 \\
\hline Sphere & $x^{2}+y^{2}+z^{2}-a^{2}=0$ & $a=9$ & {$[-10,10]^{3}$} & 0.1111 & 0.1111 \\
\hline $\begin{array}{l}\text { Rounded } \\
\text { cube }\end{array}$ & $x^{4}+y^{4}+z^{4}-a^{4}=0$ & $a=9$ & {$[-10,10]^{3}$} & 0 & 0.2822 \\
\hline $\begin{array}{l}\text { Goursat's } \\
\text { surface }\end{array}$ & $a x^{4}+a y^{4}+a z^{4}+b x^{2}+b y^{2}+b z^{2}+c=0$ & $(a, b, c)=(0.03,-2,-8)$ & {$[-10,10]^{3}$} & -0.1501 & 0.4532 \\
\hline
\end{tabular}

Table 1: Equations, parameters and domains of Euclidean shapes considered in the experimental evaluation $(t \in[0,2 \pi]$ for parametric curves). Please refer to Fig. 2 for illustrations.

convergence in $O\left(h^{\frac{1}{3}}\right)$. We first need to confirm this setting considering $\alpha_{m}$ values in $\left\{\frac{1}{2}, \frac{2}{5}, \frac{1}{3}, \frac{2}{7}, \frac{1}{4}\right\}$. Fig. 3 $\left(a, b\right.$ and $c$ ) presents results for the worst-case $\left(l_{\infty}\right)$ distance between the true expected curvature values and the estimated ones. For multigrid ellipses, we observe experimental convergence for several $\alpha_{m}$ values (except for $\alpha_{m}=\frac{1}{2}$ ). As suggested by Theorem 2, for $\alpha_{m}=\frac{1}{3}$ the behavior of the $l_{\infty}$ error is experimentally in $O\left(h^{\frac{1}{3}}\right)$. The theorem is defined in the general case, but the big $O$ error can be improved with some further hypothesis on the shape. This could explain why better convergence speeds seem to be obtained when $\alpha_{m}=\frac{2}{7}$ and $\frac{1}{4}$. For non-convex shapes (flower and accelerated flower), we still observe the convergence. Interestingly, values $\alpha_{m}$ greater than $\frac{1}{3}$ (and thus larger digital kernel size) seems to lead to slightly better estimations. The theoretical rationale behind this observation should be explored in future works.

We performed the same analysis in 3D for the mean and principal curvature estimators. Section 3 in [31] and Theorem 6 theoretically prove that $\alpha_{m}=\frac{1}{3}$ brings to an $l_{\infty}$ error at least in $O\left(h^{\frac{1}{3}}\right)$. Fig. 4 gives results of $l_{\infty}$ error for various $\alpha_{m}$ values. We observe that for both convex and non-convex shapes, $\alpha_{m}=\frac{1}{3}$ provides expected convergence speed in mean and principal curvatures estimation. As in $2 \mathrm{D}$, better convergence speed can be obtained with $\alpha_{m}=\frac{2}{7}$ and $\frac{1}{4}$.

In Fig. 3-( $d, e$ and $f)$ we compare the proposed 2D curvature estimator (II with $\alpha_{m}=\frac{1}{3}$ ) with BC, MDSS, MDCA estimators for the $l_{2}$ (mean error) and $l_{\infty}$ error metrics. In these noise-free shapes, $l_{\infty}$ convergence speeds of MDCA is close to II. We observe a convergence for BC, but with lower convergence 


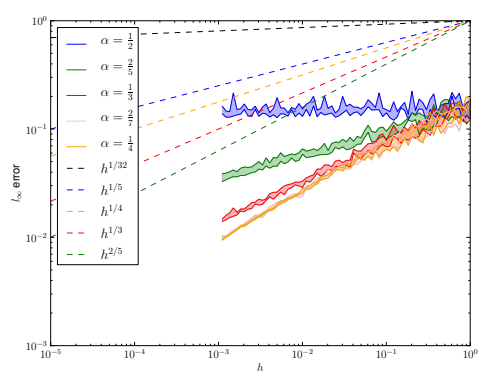

(a) Ellipse- $\hat{\kappa}_{R^{-}} \alpha_{m}$

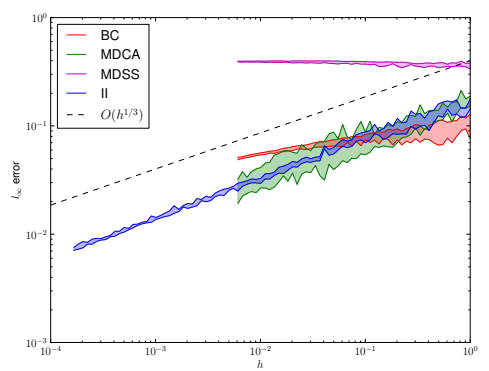

(d) Ellipse- $\hat{\kappa}_{R}-l_{\infty}$

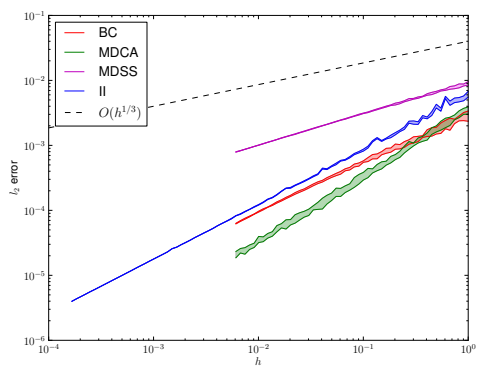

(g) Ellipse- $\hat{\kappa}_{R}-l_{2}$

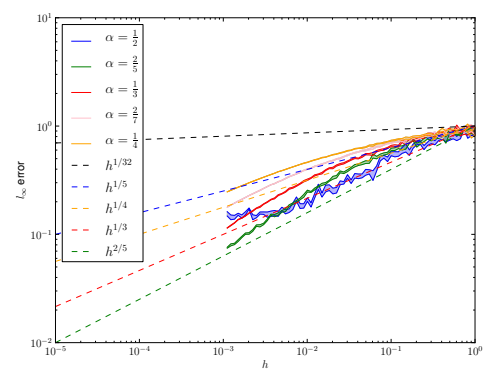

(b) Flower- $\hat{\kappa}_{R^{-}} \alpha_{m}$

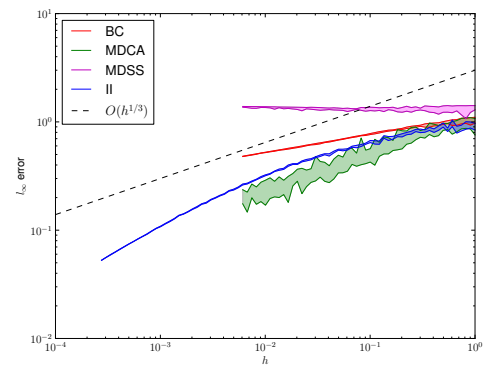

(e) Flower- $\hat{\kappa}_{R^{-}} l_{\infty}$

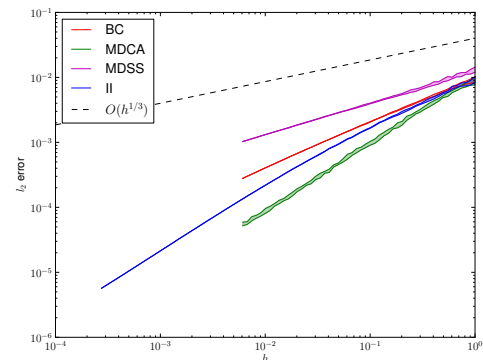

(h) Flower- $\hat{\kappa}_{R}-l_{2}$

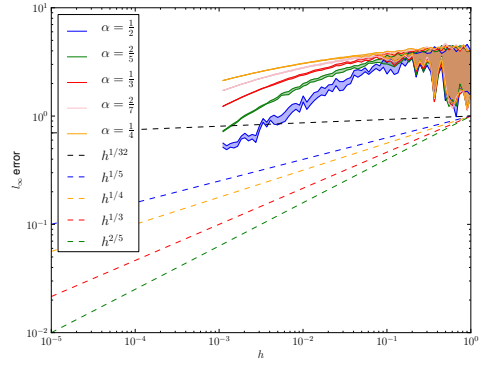

(c) AccFlower- $\hat{\kappa}_{R^{-}} \alpha_{m}$

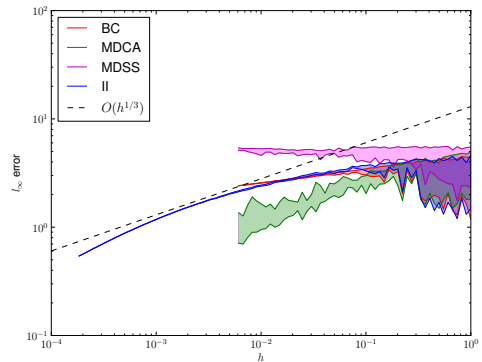

(f) AccFlower- $\hat{\kappa}_{R}-l_{\infty}$

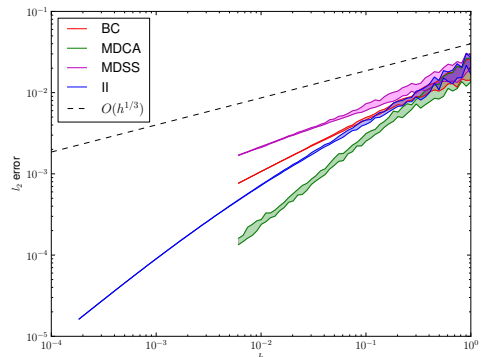

(i) AccFlower- $\hat{\kappa}_{R}-l_{2}$

Figure 3: Comparison of $h^{\alpha}$ on an ellipse $(a)$, a flower $(b)$ and an accelerated flower $(c)$. Comparison of $l_{\infty}$ and $l_{2}$ curvature error with BC [25], MDSS [42, 23] and MDCA [27] on an ellipse ( $d$ and $g$ ), a flower $(e$ and $h)$ and an accelerated flower $(f$ and i). 


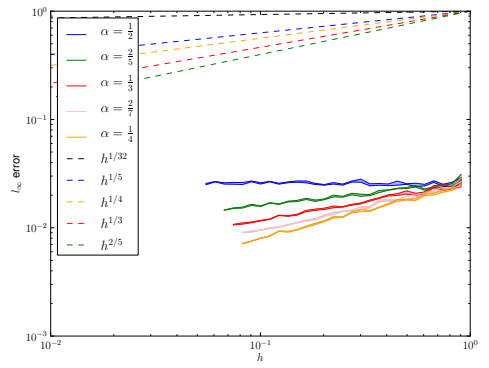

(a) Sphere- $\hat{H}_{R^{-}} \alpha_{m}$

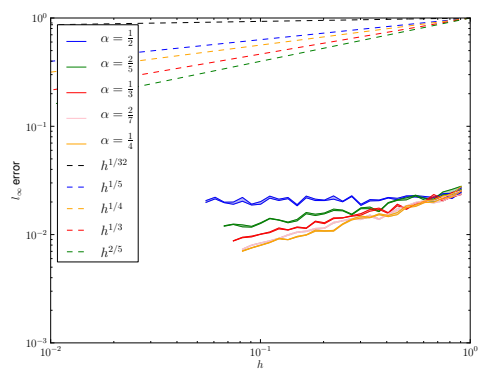

(d) Sphere- $\hat{\kappa}_{R}^{1}-\alpha_{m}$

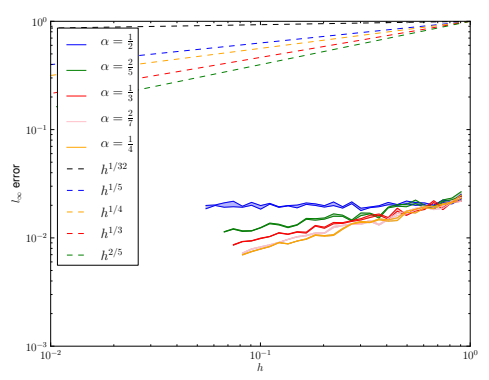

(g) Sphere- $\hat{\kappa}_{R}^{2}-\alpha_{m}$

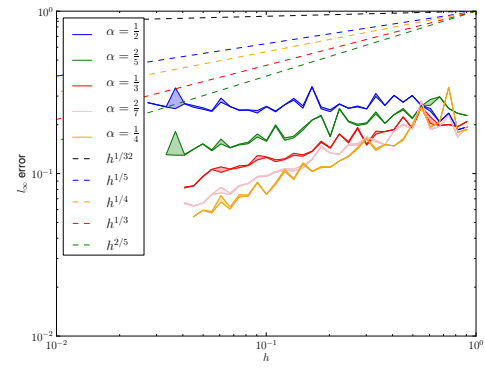

(b) RoundedCube2- $\hat{H}_{R^{-}} \alpha_{m}$

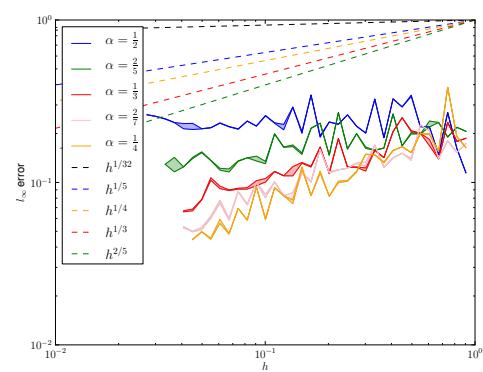

(e) RoundedCube $2-\hat{\kappa}_{R}^{1}-\alpha_{m}$

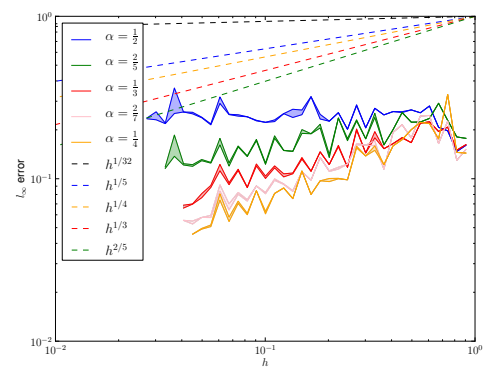

(h) RoundedCube2- $\hat{\kappa}_{R}^{2}-\alpha_{m}$

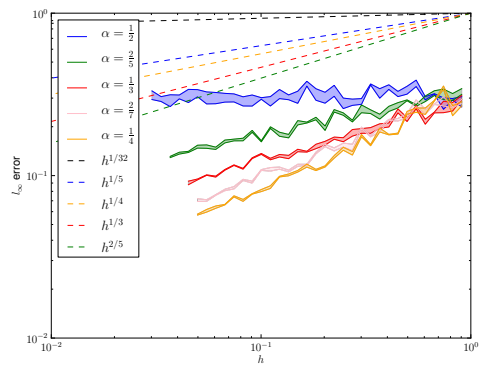

(c) Goursat- $\hat{H}_{R^{-}} \alpha_{m}$

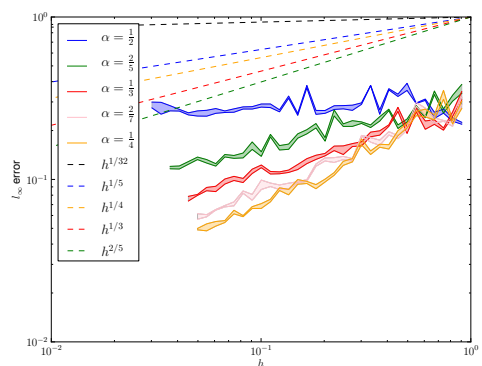

(f) Goursat- $\hat{\kappa}_{R}^{1}-\alpha_{m}$

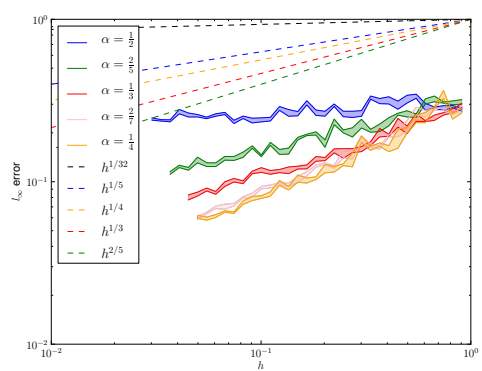

(i) Goursat- $\hat{\kappa}_{R}^{2}-\alpha_{m}$

Figure 4: Comparison of $h^{\alpha}$ for mean curvature and principal curvatures on a sphere $(a, d$ and $g)$, a rounded cube $(b, e$ and $h$ ) and Goursat's surface $(c, f$ and $i)$. 
speeds. Note that MDSS is showed experimentally non convergent. We observe for ellipses that BC provides better $l_{2}$ results than II for high $h$ values, but the behavior of both curves show that II will be better when $h$ is refined. For flower and accelerated flower, we have the same behavior for $l_{2}$ than for $l_{\infty}$ error.

In all graphs, we had to stop the computations for BC and MDCA for the following reasons: in our implementation of $\mathrm{BC}$, the mask size becomes too large for small $h$ values which induces memory usage issues. For MDCA, circular arc recognition in DGTAL is driven by a geometrical predicate based on a determinant computation of squared point coordinates. Hence, small $h$ values lead to numerical capacity issues and thus instability (which could be solved considering arbitrary precision integer numbers but would lead to efficiency issues). The proposed integral invariant estimator does not suffer from these kinds of issues. Note that for the finest experiment $h=0.00017$, digital shapes are defined in a digital domain $235295^{2}$. At this scale, the digital ellipse has 648910 contour elements.

Fig. 5 illustrates the comparison with Jet Fitting on mean and principal curvatures with $l_{\infty}$ error metric on a sphere $(a, d$ and $g)$, a rounded cube $(b, e$ and $h)$ and Goursat's surface $(c, f$ and $i)$. We notice Jet Fitting performs better on a sphere for mean and principal curvatures than our estimators. On a rounded cube or Goursat's surface and for the mean curvature, Jet Fitting has similar convergence speed than II. However, II has slightly lower $l_{\infty}$ errors. For principal curvatures, we observe that our estimator outperforms significantly principal curvatures from Jet Fitting. If we look at mean errors $\left(l_{2}\right.$ metrics $)$ in Figure 6 , similar behaviors can be observed. Note that for the finest experiment $h=0.04$, digital shapes are defined in a digital domain $500^{3}$. At this scale, the digital rounded cube object has 1125222 surface elements.

We have also evaluated the behavior of our estimators on noisy data. Given a digital binary object, our noise model consists in swapping the grid point value at $p$ with probability defined by a power law $\beta^{1+d t(p)}$ for some user-specified $\beta \in[0,1](d t(p)$ corresponds to the distance of $p$ to the boundary of the original digital shape). Such noise model, so called KANUNGo [44], is particularly well-adapted to evaluate the stability of digital geometry algorithms [45]. In Figure $7-(a, d)$, examples of noisified objects are given. Our experimental setting can be described as follows: for both the flower in $2 \mathrm{D}$ and the rounded cube in 3D ( $h=0.1$ for both), we slightly increase the noise parameter from 0 to 1 and we plot the $l_{\infty}$ error. In dimension two (Fig. 7-(e)), we observe that estimators based on geometrical object recognition (MDSS and MDCA) are highly sensitive to contour perturbations. Both BC and II are extremely robust to small noise but for $\beta$ greater than 0.5 , II outperforms significantly BC. In dimension 3, we observe that both II and Jet Fitting approaches lead to quite stable results. Indeed, some computations in the Jet Fitting approach rely on a point-set PCA (Principal Component Analysis) which also provides robust statistics.

In Fig. 8, we detail timings in logscale of various estimators on the flower object in $2 \mathrm{D}$ and the rounded cube in 3D. As expected, approaches based on object recognition in dimension 2 (MDSS and MDCA) provide faster computations. We also observe that II is a bit slower but has a asymptotic behavior much more favorable that BC. In dimension 3 (Fig. 8-(b)), we observe that Jet fitting and II behaviors are similar 


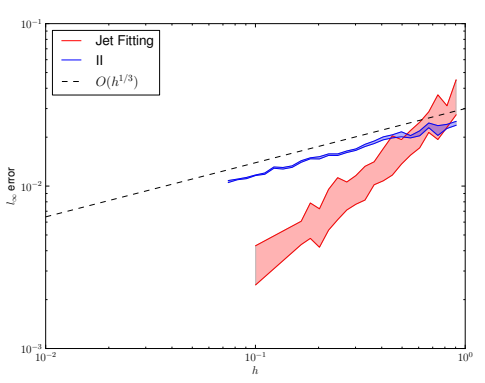

(a) Sphere- $\hat{H}_{R}-l_{\infty}$

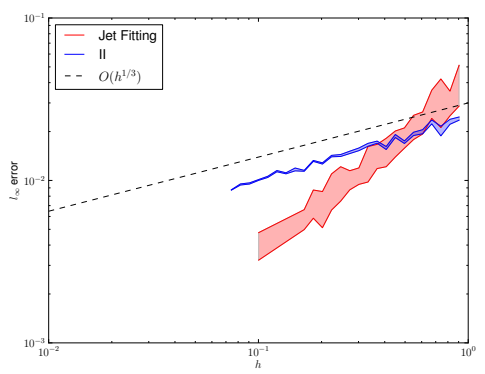

(d) Sphere- $\hat{\kappa}_{R}^{1}-l_{\infty}$

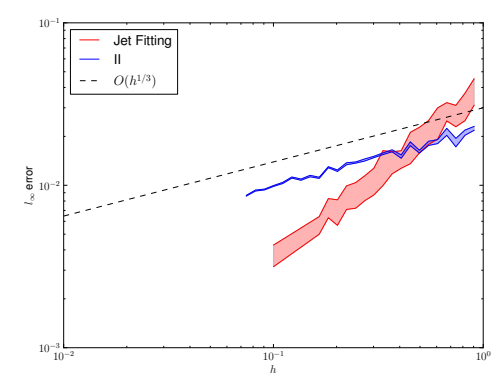

(g) Sphere- $\hat{\kappa}_{R}^{2}-l_{\infty}$

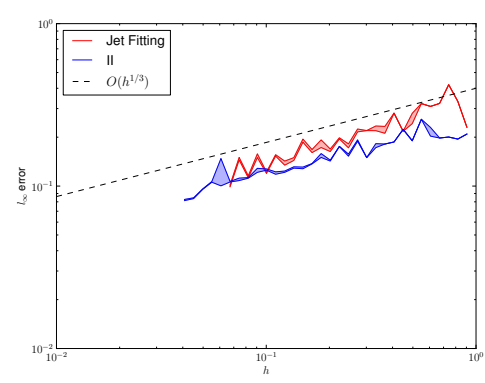

(b) RoundedCube- $\hat{H}_{R}-l_{\infty}$

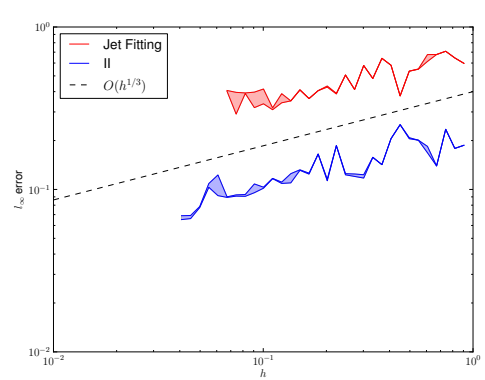

(e) RoundedCube- $\hat{\kappa}_{R}^{1} l_{\infty}$

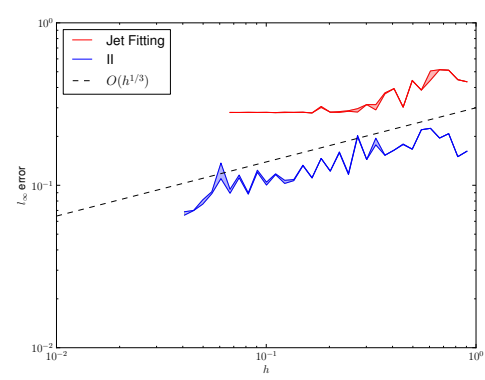

(h) RoundedCube- $\hat{\kappa}_{R}^{2}-l_{\infty}$

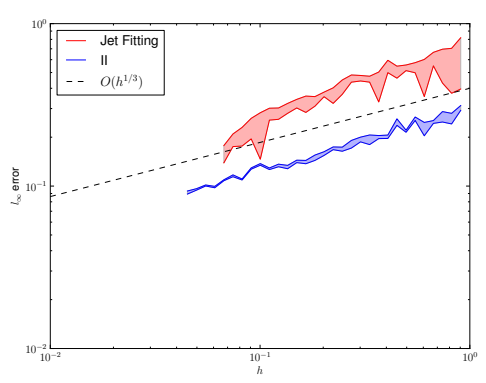

(c) Goursat- $\hat{H}_{R^{-}} l_{\infty}$

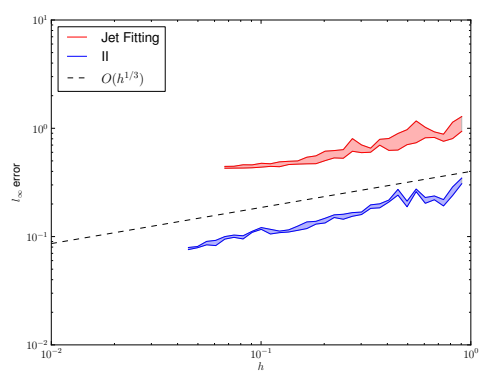

(f) Goursat- $\hat{\kappa}_{R}^{1} l_{\infty}$

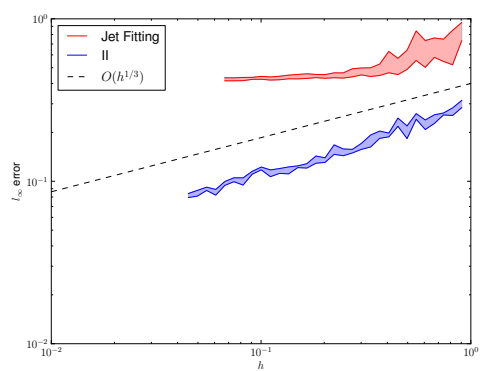

(i) Goursat- $\hat{\kappa}_{R}^{2} l_{\infty}$

Figure 5: Comparison of $l_{\infty}$ mean and principal curvatures error with Jet Fitting [16] on a sphere $(a, d$ and $g)$, a rounded cube $(b, e$ and $h)$ and Goursat's surface $(c, f$ and $i)$. 


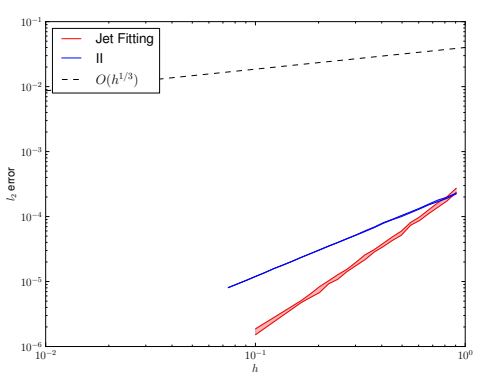

(a) Sphere- $\hat{H}_{R}-l_{2}$

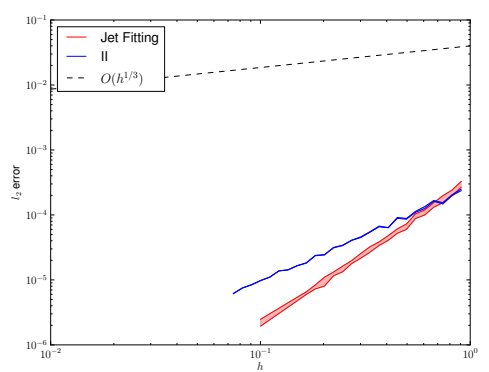

(d) Sphere- $\hat{\kappa}_{R}^{1}-l_{2}$

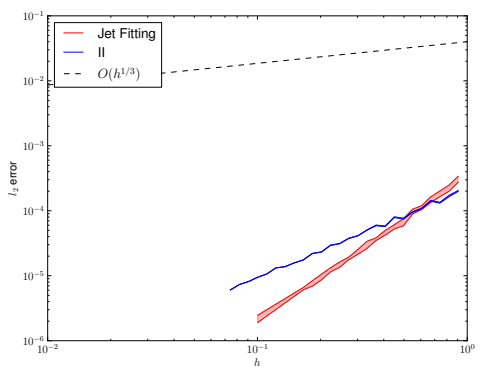

(g) Sphere- $\hat{\kappa}_{R}^{2}-l_{2}$

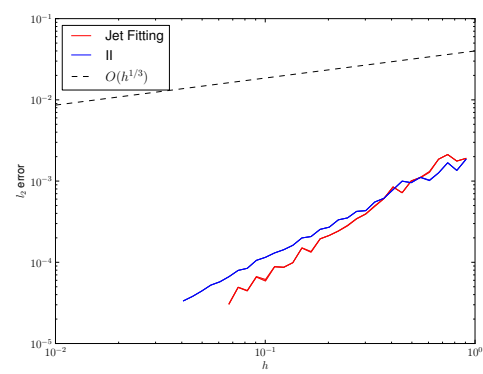

(b) RoundedCube- $\hat{H}_{R}-l_{2}$

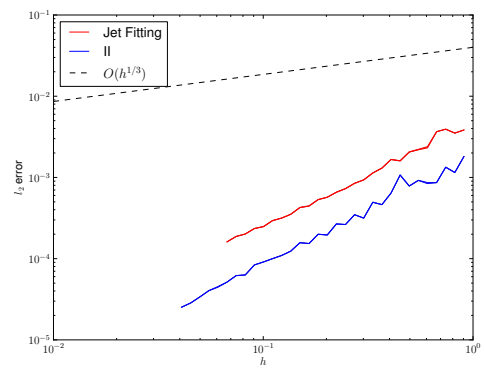

(e) RoundedCube- $\hat{\kappa}_{R}^{1}-l_{2}$

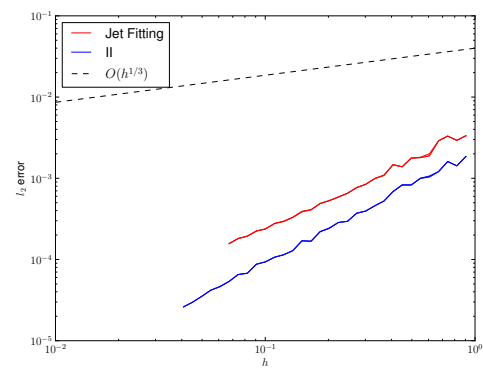

(h) RoundedCube- $\hat{\kappa}_{R}^{2}-l_{2}$

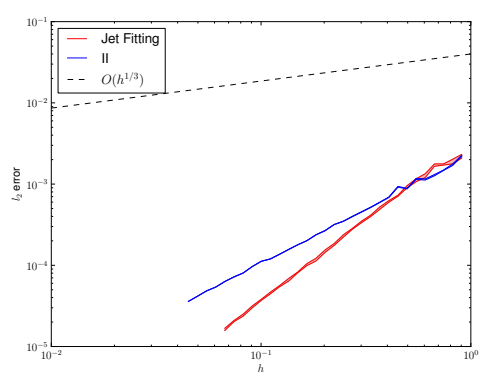

(c) Goursat- $\hat{H}_{R}-l_{2}$

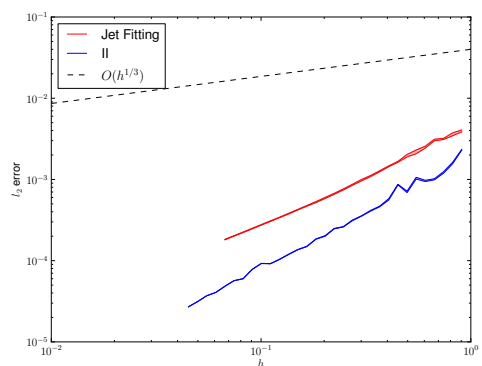

(f) Goursat- $\hat{\kappa}_{R}^{1}-l_{2}$

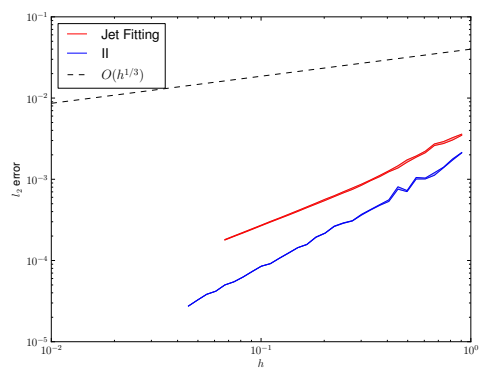

(i) Goursat- $\hat{\kappa}_{R}^{2}-l_{2}$

Figure 6: Comparison of $l_{2}$ mean and principal curvatures error with Jet Fitting [16] on a sphere ( $a, d$ and $g$ ), a rounded cube $(b, e$ and $h)$ and Goursat's surface $(c, f$, and $i)$. 


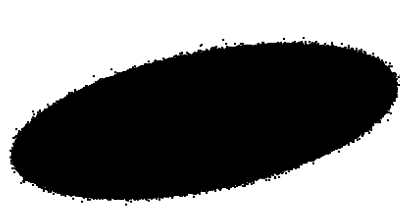

(a)

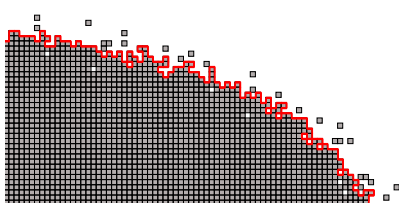

(b)

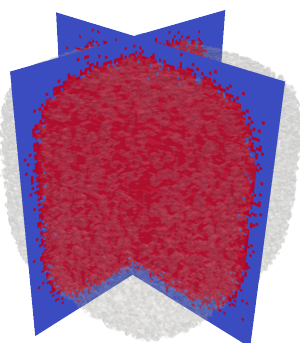

(c)

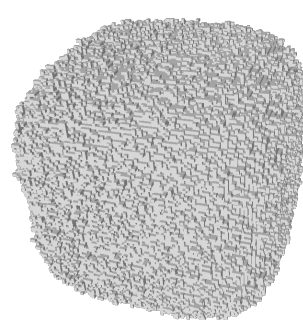

(d)

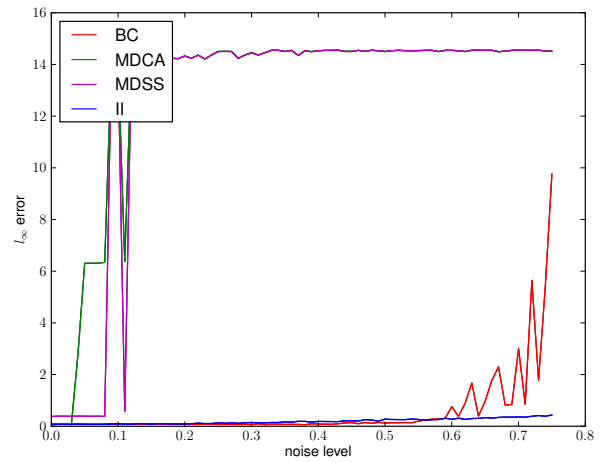

(e) Ellipse-Noise- $\hat{\kappa}_{R}-l_{\infty}$

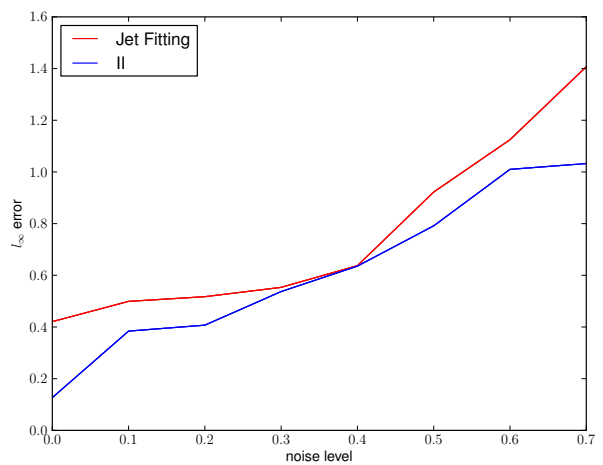

(g) Ellipsoid-Noise- $\hat{\kappa}_{R}^{1}-l_{\infty}$

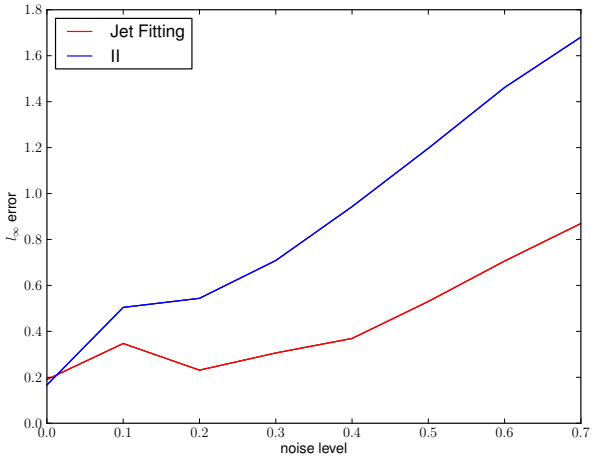

(f) Ellipsoid-Noise- $\hat{H}_{R}-l_{\infty}$

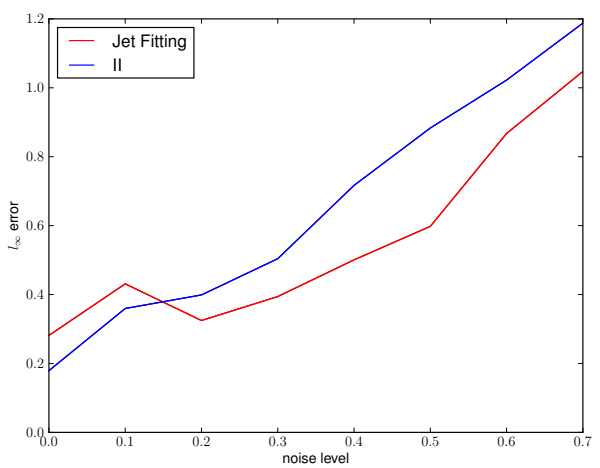

(h) Ellipsoid-Noise- $\hat{\kappa}_{R}^{2}-l_{\infty}$

Figure 7: Ellipse with $\beta=0.5(a)$ and a zoom of his boundary in red $(b)$. Rounded cube with $\beta=0.5(c)$ and his boundary $(d)$. Comparison of $2 \mathrm{D}$ estimators on different level of noise on an ellipse $(e)$. Comparison of 3D estimators for mean $(f)$ and principal curvatures $(g$ and $h$ ) on different level of noise on a rounded cube. 


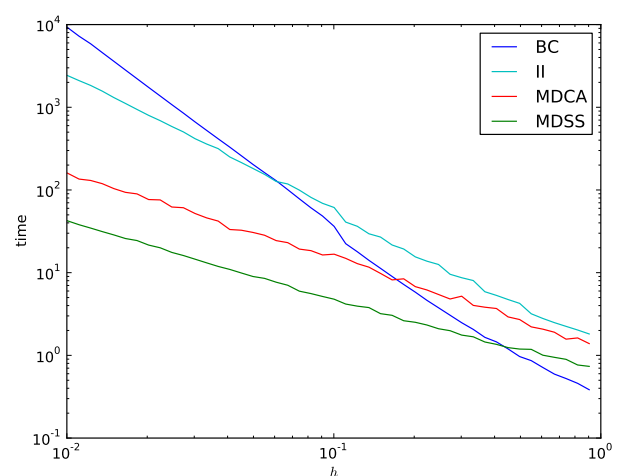

(a)

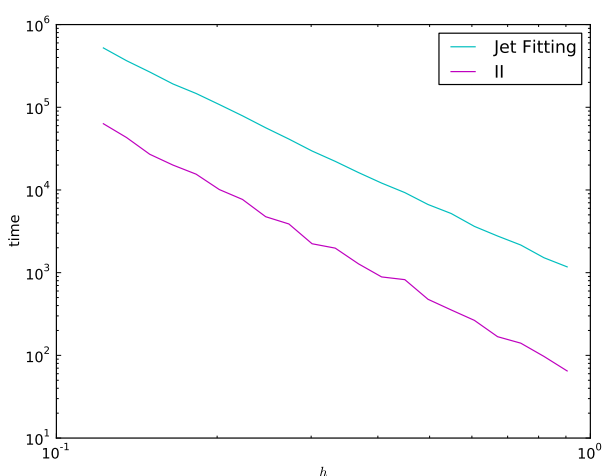

(b)

Figure 8: Timings in milliseconds for $2 \mathrm{D}$ estimators on a flower $(a)$ and $3 \mathrm{D}$ estimators on a rounded cube $(b)$. Results have been obtained on a Intel Xeon $2.27 \mathrm{GHz}$ desktop machine.

and that II is 10 times faster than Jet fitting.

Finally, Fig. 9 shows mean curvature and principal directions mapped on various shapes (rounded cube, Goursat's surface, Leopold surface) and on an object (Stanford bunny). From these experiments, we can see that principal directions are nicely captured by covariance matrix eigenvectors.

\section{Conclusion}

In this paper, we have used integral invariant results from differential geometry to design simple and efficient digital curvature estimators in dimension 2 and 3. Digital Geometry is a perfect domain for such differential tools: volume, area or geometrical moments computations are digital by nature, interesting connections to fundamental results on Gauss digitization exist, fast computations are induced by the specific geometry of digital surfaces.

For curvature estimation in dimension 2 as well as principal curvature estimations in dimension 3, we have proven a theoretical uniform convergence in $O\left(h^{\frac{1}{3}}\right)$ for $C^{3}$ smooth object boundaries. Experimental evaluation has not only confirmed this bound but has also shown that these estimators can be computed efficiently in practice with low computational costs. Our digital Integral Invariant estimators and all other estimators used in the experimental evaluation section are publicly available in DGTAL [32].

Convergence speed are obtained with a weak constraint on the distance between $\hat{x}$ and $x$ (which just needs to be lower that $h$ for the $l_{\infty}$ metric). Using a specific projection as discussed in [33], better convergence speed is expected at least for dimension 2 . 


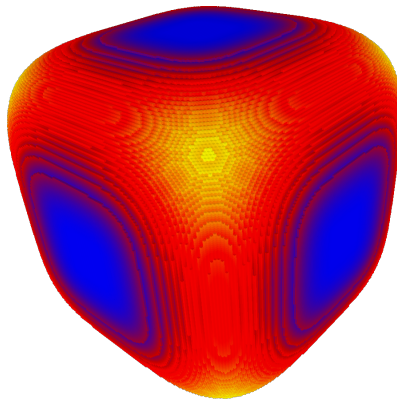

(a)

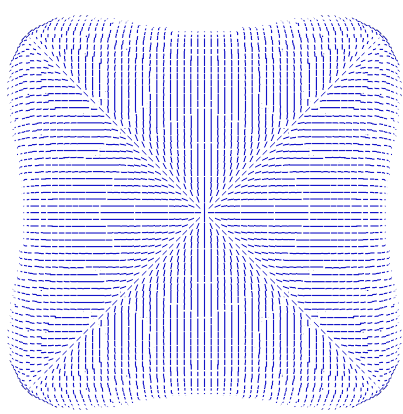

(e)

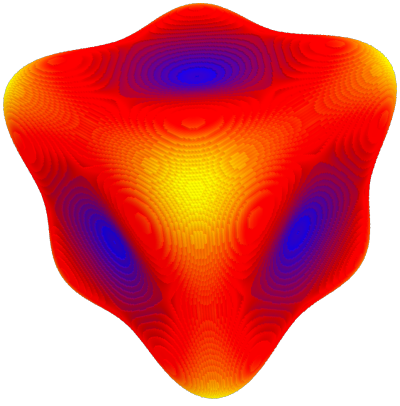

(b)

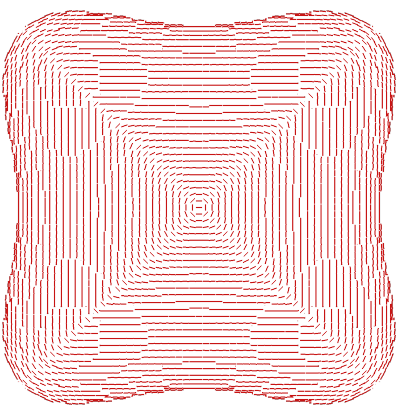

(f)

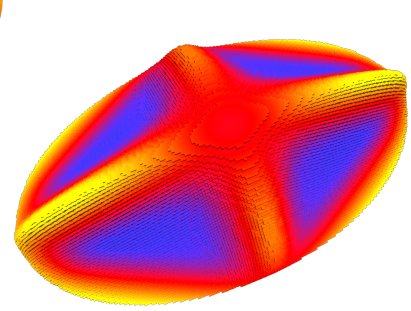

(c)

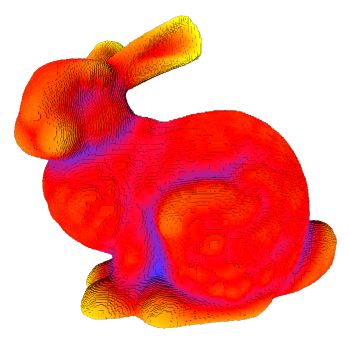

(d)

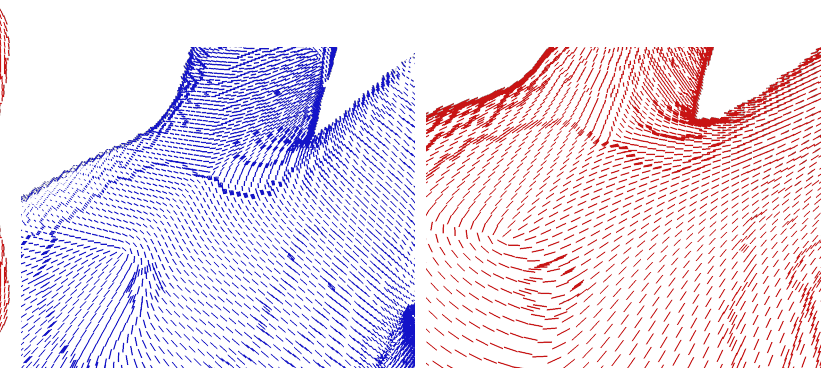

(g)

(h)

Figure 9: Illustration of 3D curvature estimation. Mean curvature on rounded cube $(a)$, Goursat's surface $(b)$, Leopold surface $(c)$ and a bunny $(d)$. First principal direction and second principal direction Goursat's surface $(e$ and $f$ ) and Stanford bunny $(g$ and $h)$. 


\section{Acknowledgment}

This work has been mainly funded by DigitalSnow ANR-11-BS02-009 research grants. We gratefully acknowledge support from the CNRS/IN2P3 Computing Center (Lyon/Villeurbanne - France), for providing a significant amount of the computing resources needed for this work. We also thank the Stanford 3D Scanning Repository for Bunny model.

\section{Appendix A. Technical lemmas}

Appendix A.1. Technical lemmas related to covariance matrices and continuous moments

We gather here a few technical facts and results related to covariance matrices, digital and continuous $(p, q, s)$-moments.

Lemma 4. Translation invariance for covariance matrix:

- for any finite subset $Y \subset \mathbb{R}^{3}$, for any vector $\mathbf{v} \in \mathbb{R}^{3}, J(Y+\mathbf{v})=J(Y)$.

- for any finite subset $Z \subset \mathbb{Z}^{3}$, for any integral vector $\mathbf{v} \in \mathbb{Z}^{3}$, for any $h>0, \hat{J}_{h}(Z+\mathbf{v})=\hat{J}_{h}(Z)$.

Lemma 5. Let $B_{R}(t)$ be the ball of radius $R$ and center $t$. Then, for any non-empty $Y \subset B_{R}(t)$,

$$
\begin{aligned}
& m_{0,0,0}(Y)=O\left(R^{3}\right), \\
& m_{p, q, s}(Y)=O\left(R^{3}\left(\|t\|_{\infty}+R\right)\right), \quad(p+q+s=1) \\
& m_{p, q, s}(Y)=O\left(R^{3}\left(\|t\|_{\infty}^{2}+R\|t\|_{\infty}+R^{2}\right)\right), \quad(p+q+s=2)
\end{aligned}
$$

and

$$
m_{p, q, s}(Y) / m_{0,0,0}(Y)=O(R+t), \quad(p+q+s=1)
$$

Proof. Eq.(A.1) is immediate since 0-order moment is the volume of $Y$, hence it cannot exceed the volume of the ball which is $\frac{4}{3} \pi R^{3}$. For Eq.(A.2), we make the change of variable $\left(x^{\prime}, y^{\prime}, z^{\prime}\right)=(x, y, z)-t$ in the following expression:

$$
\begin{aligned}
m_{1,0,0}(Y) & =\iiint_{Y} x d x d y d z=\iiint_{Y-t}\left(x^{\prime}+t_{x}\right) d x^{\prime} d y^{\prime} d z^{\prime} \\
& =t_{x} \operatorname{Vol}(Y)+m_{1,0,0}(Y-t) .
\end{aligned}
$$

In the first term, $\operatorname{Vol}(Y)$ is bounded by the volume of any ball of radius $R$. By the additivity of integrals, the second term is maximized by the $(1,0,0)$-moment of the half-ball $B_{R}^{+}(0)$ centered on 0 and lying in the 


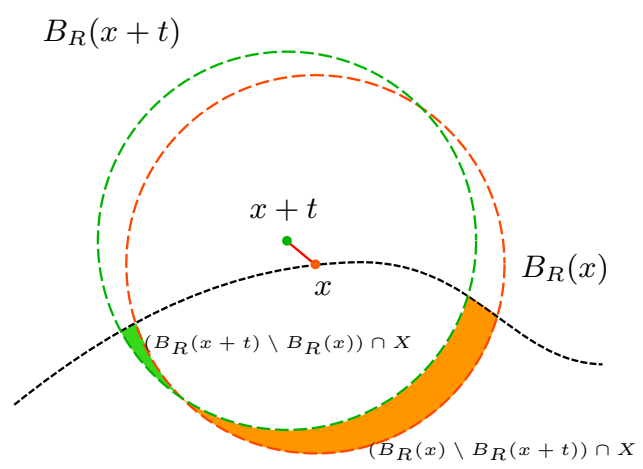

(a)

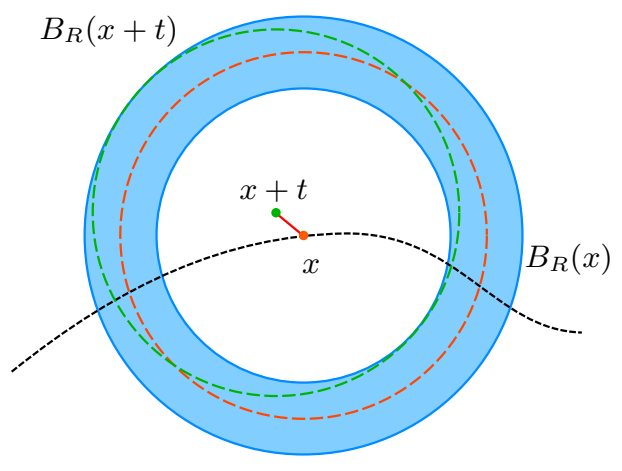

(b)

Figure A.10: Illustration for Lemma 3.

positive $x$ orthants. Now, using spherical coordinates, we get

$$
\begin{aligned}
m_{1,0,0}\left(B_{R}^{+}(0)\right) & =\int_{0}^{R} \int_{\frac{-\pi}{2}}^{\frac{\pi}{2}} \int_{\frac{-\pi}{2}}^{\frac{\pi}{2}}(\rho \cos \phi \cos \theta)\left(\rho^{2} \cos \phi\right) d \theta d \phi d \rho \\
& =\left[\frac{\rho^{4}}{4}\right]_{0}^{R}[\sin \theta]_{-\frac{\pi}{2}}^{\frac{\pi}{2}} \frac{1}{2}[\phi+\sin (\phi) \cos (\phi)]_{-\frac{\pi}{2}}^{\frac{\pi}{2}} \\
& =\frac{\pi}{4} R^{4},
\end{aligned}
$$

which concludes for Eq.(A.2) (other cases $p+q+s=1$ are similar). Other equations are proved in a similar way.

\section{Appendix A.2. Proof of lemma 3}

Proof. By decomposing $X$ according to the two shifted balls (see Fig. A.10), we get:

$$
\begin{aligned}
m_{p, q, s}\left(B_{R}(x+\mathbf{t}) \cap X\right)-m_{p, q, s}\left(B_{R}(x) \cap X\right) & =m_{p, q, s}\left(\left(B_{R}(x+\mathbf{t}) \backslash B_{R}(x)\right) \cap X\right) \\
& -m_{p, q, s}\left(\left(B_{R}(x) \backslash B_{R}(x+\mathbf{t})\right) \cap X\right)
\end{aligned}
$$

We denote by $\Delta(\mathbf{t})$ the difference on the right term of preceding equation. The two sets on the right form the symmetric difference (symbol $\ominus$ ) of the two shifted balls. We will use the following fact:

$$
\emptyset \neq Y_{1} \subset Y_{2} \subset \mathbb{R}^{3} \Rightarrow\left|\sup _{Y \subset Y_{1}} m_{p, q, s}(Y)\right| \leq\left|\sup _{Y \subset Y_{2}} m_{p, q, s}(Y)\right| .
$$

By additivity of integrals, we have immediately:

$$
\begin{aligned}
|\Delta(\mathbf{t})| & =\left|m_{p, q, s}\left(\left(B_{R}(x+\mathbf{t}) \ominus B_{R}(x)\right) \cap X\right)\right| \\
& \leq \sup _{Y \subset\left(B_{R}(x+\mathbf{t}) \ominus B_{R}(x)\right) \cap X}\left|m_{p, q, s}(Y)\right| \\
& \leq \sup _{Y \subset B_{R}(x+\mathbf{t}) \ominus B_{R}(x)}\left|m_{p, q, s}(Y)\right| \\
& \leq \sup _{Y \subset B_{R+t}(x)-B_{R-t}(x)}\left|m_{p, q, s}(Y)\right| .
\end{aligned}
$$


In the last row, we cover the symmetric difference of two shifted balls by the difference of two balls of same center, a kind of spherical shell or annulus in 2D (see Fig. A.10). Although it is a rough upper bound, it induces the same order of perturbation. We denote this set $B_{R+t}(x)-B_{R-t}(x)$ by $H_{R, t}(x)$.

For zeroth order moment, we use simply the volume of the ball:

$$
\begin{aligned}
\sup _{Y \subset H_{R, t}(x)}\left|m_{0,0,0}(Y)\right| & =m_{0,0,0}\left(H_{R, t}(x)\right) \\
& =\frac{4 \pi}{3}\left(6 R^{2} t+2 t^{3}\right)=O\left(t R^{2}\right) .
\end{aligned}
$$

For first order moment, we translate the shape to the origin, then we use the previous result plus the fact that the centered 1,0,0-moment is maximized by the $x$-positive half-ball:

$$
\begin{aligned}
\sup _{Y \subset H_{R, t}(x)}\left|m_{1,0,0}(Y)\right| & \leq \sup _{Y \subset H_{R, t}(0)}\left|m_{1,0,0}(Y)\right|+\left|x_{x}\right|\left|m_{0,0,0}(Y)\right| \\
& =m_{1,0,0}\left(B_{R+t}^{+}(0)-B_{R-t}^{+}(0)\right)+O\left(\left|x_{x}\right| t R^{2}\right) \\
& =2 \pi\left(R^{3} t+R t^{3}\right)+O\left(\left|x_{x}\right| t R^{2}\right) \\
& =O\left(t R^{3}\right)+O\left(\|x\| t R^{2}\right)
\end{aligned}
$$

For second order moment, we translate the shape to the origin, then we use the two previous results plus the fact that the 2,0,0-moment is maximized by the ball:

$$
\begin{aligned}
\sup _{Y \subset H_{R, t}(x)}\left|m_{2,0,0}(Y)\right| & \leq \sup _{Y \subset H_{R, t}(0)}\left|m_{2,0,0}(Y)\right|+2\left|x_{x} \| m_{1,0,0}(Y)+x_{x}^{2}\right| m_{0,0,0}(Y) \mid \\
& =m_{2,0,0}\left(H_{R, t}(0)\right)+\|x\|\left(O\left(t R^{3}\right)+O\left(\|x\| t R^{2}\right)\right)+x_{x}^{2} O\left(t R^{2}\right) \\
& =O\left(t R^{4}\right)+O\left(\|x\| t R^{3}\right)+O\left(\|x\|^{2} t R^{2}\right)
\end{aligned}
$$

Other moments are proved similarly.

\section{References}

[1] R. Klette, A. Rosenfeld, Digital Geometry: Geometric Methods for Digital Picture Analysis, Series in Computer Graphics and Geometric Modelin, Morgan Kaufmann, 2004.

[2] D. Coeurjolly, J.-O. Lachaud, T. Roussillon, Digital Geometry Algorithms, Theoretical Foundations and Applications of Computational Imaging, volume 2 of $L N C V B$, Springer, 2012, pp. 395-424.

[3] N. Amenta, M. Bern, M. Kamvysselis, A new voronoi-based surface reconstruction algorithm, in: Proceedings of the 25th annual conference on Computer graphics and interactive techniques, ACM, 1998, pp. 415-421.

[4] Q. Mérigot, M. Ovsjanikov, L. Guibas, Voronoi-based curvature and feature estimation from point clouds, Visualization and Computer Graphics, IEEE Transactions on 17 (2011) 743-756.

[5] T. Surazhsky, E. Magid, O. Soldea, G. Elber, E. Rivlin, A comparison of gaussian and mean curvatures estimation methods on triangular meshes, in: Robotics and Automation, 2003. Proceedings. ICRA '03. IEEE International Conference on, volume 1, 2003, pp. 1021-1026. 
[6] T. D. Gatzke, C. M. Grimm, Estimating curvature on triangular meshes, International Journal of Shape Modeling 12 (2006) 1-28.

[7] M. Desbrun, A. N. Hirani, M. Leok, J. E. Marsden, Discrete exterior calculus, arXiv preprint math/0508341 (2005).

[8] A. I. Bobenko, Y. B. Suris, Discrete differential geometry: Integrable structure, volume 98, AMS Bookstore, 2008.

[9] D. L. Page, Y. Sun, A. F. Koschan, J. Paik, M. A. Abidi, Normal vector voting: Crease detection and curvature estimation on large, noisy meshes, Graphical Models 64 (2002) 199-229.

[10] S. Rusinkiewicz, Estimating curvatures and their derivatives on triangle meshes, in: 3D Data Processing, Visualization and Transmission, 2004. 3DPVT 2004. Proceedings. 2nd International Symposium on, 2004, pp. 486-493.

[11] G. Xu, Convergence analysis of a discretization scheme for gaussian curvature over triangular surfaces, Computer Aided Geometric Design 23 (2006) 193-207.

[12] D. Cohen-Steiner, J.-M. Morvan, Restricted delaunay triangulations and normal cycle, in: Proceedings of the nineteenth annual symposium on Computational geometry, SCG'03, ACM, New York, NY, USA, 2003, pp. 312-321. URL: http://doi.acm.org/10.1145/777792.777839.

[13] D. Cohen-Steiner, J.-M. Morvan, Second fundamental measure of geometric sets and local approximation of curvatures, Journal of Differential Geometry 74 (2006) 363-394.

[14] H. Pottmann, J. Wallner, Y. Yang, Y. Lai, S. Hu, Principal curvatures from the integral invariant viewpoint, Computer Aided Geometric Design 24 (2007) 428-442.

[15] H. Pottmann, J. Wallner, Q. Huang, Y. Yang, Integral invariants for robust geometry processing, Computer Aided Geometric Design 26 (2009) 37-60.

[16] F. Cazals, M. Pouget, Estimating differential quantities using polynomial fitting of osculating jets, Computer Aided Geometric Design 22 (2005) 121-146.

[17] P. Alliez, D. Cohen-Steiner, Y. Tong, M. Desbrun, Voronoi-based variational reconstruction of unoriented point sets, in: Symposium on Geometry processing, volume 7, 2007, pp. 39-48.

[18] Q. Mérigot, M. Ovsjanikov, L. Guibas, Robust voronoi-based curvature and feature estimation, in: 2009 SIAM/ACM Joint Conference on Geometric and Physical Modeling, SPM'09, ACM, New York, NY, USA, 2009, pp. 1-12. URL: http://doi.acm.org/10.1145/1629255.1629257.

[19] B. Li, R. Schnabel, R. Klein, Z. Cheng, G. Dang, S. Jin, Robust normal estimation for point clouds with sharp features, Computers \& Graphics 34 (2010) 94-106.

[20] A. Boulch, R. Marlet, Fast and robust normal estimation for point clouds with sharp features, Computer Graphics Forum 31 (2012) 1765-1774.

[21] J. Zhang, J. Cao, X. Liu, J. Wang, J. Liu, X. Shi, Point cloud normal estimation via low-rank subspace clustering, Computers \& Graphics 37 (2013) 697-706.

[22] D. Coeurjolly, R. Klette, A comparative evaluation of length estimators of digital curves, IEEE Trans. on Pattern Analysis and Machine Intelligence 26 (2004) 252-258.

[23] F. de Vieilleville, J.-O. Lachaud, F. Feschet, Maximal digital straight segments and convergence of discrete geometric estimators, Journal of Mathematical Image and Vision 27 (2007) 471-502.

[24] R. Malgouyres, F. Brunet, S. Fourey, Binomial convolutions and derivatives estimation from noisy discretizations, in: Discrete Geometry for Computer Imagery, volume 4992 of LNCS, Springer, 2008, pp. 370-379.

[25] H.-A. Esbelin, R. Malgouyres, C. Cartade, Convergence of binomial-based derivative estimation for 2 noisy discretized curves, Theoretical Computer Science 412 (2011) 4805 - 4813.

[26] L. Provot, Y. Gérard, Estimation of the derivatives of a digital function with a convergent bounded error, in: Discrete Geometry for Computer Imagery, LNCS, Springer, 2011, pp. 284-295.

[27] T. Roussillon, J.-O. Lachaud, Accurate curvature estimation along digital contours with maximal digital circular arcs, in: 
Combinatorial Image Analysis, volume 6636, Springer, 2011, pp. 43-55.

[28] B. Kerautret, J.-O. Lachaud, Curvature estimation along noisy digital contours by approximate global optimization, Pattern Recognition 42 (2009) 2265 - 2278.

[29] A. Lenoir, Fast estimation of mean curvature on the surface of a 3d discrete object, in: E. Ahronovitz, C. Fiorio (Eds.), Proc. Discrete Geometry for Computer Imagery (DGCI'97), volume 1347 of Lecture Notes in Computer Science, Springer Berlin Heidelberg, 1997, pp. 175-186. URL: http://dx.doi.org/10.1007/BFb0024839.

[30] S. Fourey, R. Malgouyres, Normals and curvature estimation for digital surfaces based on convolutions, in: Discrete Geometry for Computer Imagery, LNCS, Springer, 2008, pp. 287-298.

[31] D. Coeurjolly, J. L. Lachaud, J. Levallois, Integral based curvature estimators in digital geometry, in: Discrete Geometry for Computer Imagery, number 7749 in LNCS, Springer, 2013, pp. 215-227.

[32] DGtal: Digital geometry tools and algorithms library, http://libdgtal.org.

[33] J.-O. Lachaud, Espaces non-euclidiens et analyse d'image : modèles déformables riemanniens et discrets, topologie et géométrie discrète, Habilitation à diriger des recherches, Université Bordeaux 1, Talence, France, 2006.

[34] J. W. Bullard, E. J. Garboczi, W. C. Carter, E. R. Fullet, Numerical methods for computing interfacial mean curvature, Computational materials science 4 (1995) 103-116.

[35] E. Krätzel, Lattice points, volume 33, Springer, 1988.

[36] M. N. Huxley, Area, lattice points and exponential sums, Oxford Science publications, 1996.

[37] R. Klette, J. Žunić, Multigrid convergence of calculated features in image analysis, Journal of Mathematical Imaging and Vision 13 (2000) 173-191.

[38] E. Krätzel, W. G. Nowak, Lattice points in large convex bodies, Monatshefte für Mathematik 112 (1991) 61-72.

[39] W. Müller, Lattice points in large convex bodies, Monatshefte für Mathematik 128 (1999) 315-330.

[40] G. W. Stewart, J.-g. Sun, Matrix perturbation theory (1990).

[41] R. Bhatia, Matrix analysis, volume 169, Springer, 1997.

[42] D. Coeurjolly, S. Miguet, L. Tougne, Discrete curvature based on osculating circle estimation, in: 4th International Workshop on Visual Form, volume 2059 of Lecture Notes in Computer Science, 2001, pp. 303-312.

[43] CGAL: Computational geometry algorithms library, http://www.cgal.org.

[44] T. Kanungo, Document degradation models and a methodology for degradation model validation, Ph.D. thesis, University of Washington, 1996.

[45] B. Kerautret, J. Lachaud, Meaningful scales detection along digital contours for unsupervised local noise estimation, IEEE transactions on pattern analysis and machine intelligence 34 (2012) 2379-2392. 